\title{
Granulocyte colony-stimulating factor improves left ventricular function of doxorubicin-induced cardiomyopathy
}

\author{
Longhu Li ${ }^{1,2}$, Genzou Takemura ${ }^{1}$, Yiwen $\mathrm{Li}^{1}$, Shusaku Miyata ${ }^{1}$, Masayasu Esaki ${ }^{1}$, Hideshi Okada ${ }^{1}$, \\ Hiromitsu Kanamori ${ }^{1}$, Atsushi Ogino ${ }^{1}$, Rumi Maruyama ${ }^{1}$, Munehiro Nakagawa ${ }^{1}$, Shinya Minatoguchi ${ }^{1}$, \\ Takako Fujiwara ${ }^{3}$ and Hisayoshi Fujiwara ${ }^{1}$
}

It is not well-known yet how granulocyte colony-stimulating factor (G-CSF) affects nonischemic cardiomyopathy, though its beneficial effects on acute myocardial infarction are well-established. We hypothesize that G-CSF beneficially might affect nonischemic cardiomyopathy through the direct cardioprotective effects. Here, we show that a single injection of doxorubicin (DOX, $15 \mathrm{mg} / \mathrm{kg}$ ) induced left ventricular dilatation and dysfunction in mice within 2 weeks, and that these effects were significantly attenuated by human recombinant G-CSF (100 $\mu \mathrm{g} / \mathrm{kg} /$ day for 5 days). G-CSF also protected hearts against DOX-induced cardiomyocyte atrophy/degeneration, fibrosis, inflammatory cell infiltration and down regulation of GATA-4 and sarcomeric proteins, myosin heavy chain, troponin I and desmin, both in vivo and in vitro. Cardiac cyclooxygenase-2 was upregulated and G-CSF receptor was downregulated in DOX-induced cardiomyopathy, but both of those effects were largely reversed by G-CSF. No DOX-induced apoptotic effects were seen, nor were there any changes in tumor necrosis factor- $\alpha$ or transforming growth factor- $\beta 1$ levels. Among downstream mediators of G-CSF receptor signaling, DOX-induced cardiomyopathy involved inactivation of extracellular signal-regulated protein kinase (ERK); the ERK inactivation was reversed by G-CSF. Inhibition of ERK activation, but not cyclooxygenase-2 inhibition, completely abolished beneficial effect of G-CSF on cardiac function. G-CSF did not promote differentiation of bone marrow cells into cardiomyocytes according to the experiment using green fluorescent protein-chimeric mice, and inhibition of CXCR4 ${ }^{+}$ cell homing using AMD3100 did not diminish the effect of G-CSF. Finally, G-CSF was also effective when administered after cardiomyopathy was established. In conclusion, these findings imply the therapeutic usefulness of G-CSF mainly through restoring ERK activation against DOX-induced nonischemic cardiomyopathy.

Laboratory Investigation (2007) 87, 440-455. doi:10.1038/labinvest.3700530; published online 5 March 2007

KEYWORDS: cardiomyopathy; cytokines; doxorubicin; ERK; G-CSF; heart failure

The antineoplastic drug doxorubicin (DOX) is effective in the treatment of a broad spectrum of hematogenous and solid human malignancies, but its clinical use is limited because of its adverse side effects: irreversible degenerative cardiomyopathy and congestive heart failure. ${ }^{1,2}$ The efficacy of DOX as a cytotoxic agent for the treatment of human tumors has prompted a search to find treatments that reduce or prevent the risk of cardiomyopathy and congestive heart failure. ${ }^{3-5}$ So far, however, the ability of these treatments to protect the heart from DOX-induced damage has been limited.
Recent investigations revealed unexpected effects of hematopoietic cytokines on DOX-induced cardiotoxicity; we reported erythropoietin, ${ }^{6}$ whereas another group reported thrombopoietin, ${ }^{7}$ prevented cardiac dysfunction in DOXinduced cardiomyopathy in mice. More recently, Hou et al reported preventive effects of granulocyte colony-stimulating factor (G-CSF) on progressive worsening of cardiac function in DOX-induced cardiomyopathy in rats. ${ }^{8}$ G-CSF has been applied to ischemic heart diseases, originally being expected to contribute to tissue regeneration because G-CSF can

\footnotetext{
${ }^{1}$ Second Department of Internal Medicine, Gifu University School of Medicine, Gifu, Japan; ${ }^{2}$ Cardiovascular Department of Internal Medicine, The First Affiliated Hospital of Dalian Medical University, Dalian, China and ${ }^{3}$ Department of Food Science, Kyoto Women's University, Kyoto, Japan Correspondence: Dr G Takemura, MD, PhD, Second Department of Internal Medicine, Gifu University School of Medicine, 1-1 Yanagido, Gifu 501-1194, Japan. E-mail: gt@cc.gifu-u.ac.jp

Received 06 September 2006; revised 18 December 2006; accepted 12 January 2007
} 
mobilize bone marrow-derived stem cells into peripheral blood. ${ }^{9,10}$ Recent studies have confirmed the effectiveness of G-CSF on ischemic heart diseases and revealed new mechanisms for the effect other than tissue regeneration, that is, acceleration of healing and direct tissue protection. ${ }^{11-15}$ On the other hand, there has been only one study reporting benefits of G-CSF on nonischemic cardiomyopathy, ${ }^{8}$ and thus little is known on the mechanisms for G-CSF to exert such effects. It would be much worthy to confirm the beneficial effects of G-CSF on DOX-induced cardiomyopathy in particular because of the clinical reason; G-CSF is already accepted for clinical use in combination with DOX to DOXinduced myelosuppression. Thus, if truly effective, G-CSF would be very easily translational to management of DOXinduced cardiomyopathy. In the present study, we examined the effects of G-CSF, both preventive and therapeutic, on DOX-induced cardiomyopathy and investigated the specific mechanisms underlying those effects.

\section{MATERIALS AND METHODS Experimental Protocols}

This study was approved by our Institutional Animal Research Committee and conformed to the animal care guidelines of the American Physiological Society. Cardiomyopathy was induced in male 10 -week-old C57BL/6J mice (Chubu Kagaku) with a single intraperitoneal injection of doxorubicin hydrochloride (Kyowa Hakko) at the dose of $15 \mathrm{mg} / \mathrm{kg}$ in saline. In sham-treated mice, the same volume of saline was injected in a similar manner. Recombinant human G-CSF (Chugai Pharmaceutical Co) was administered subcutaneously at a dose of $100 \mu \mathrm{g} / \mathrm{kg} /$ day in saline, once a day from day 1 to day 5 . We determined the dose of G-CSF $(100 \mu \mathrm{g} / \mathrm{kg})$ based on previous studies reporting cardioprotective effects of G-CSF. ${ }^{13,14}$ Although this dosage is extremely higher than the clinical dosage to humans (lower than $10 \mu \mathrm{g} /$ day), it is generally believed that mice have a low sensitivity to human G-CSF as presumed by the unexpectedly poor increase in granulocytes. Untreated control groups were given the same volume of saline. G-CSF was administered either prophylactically or after establishment of DOX-induced cardiomyopathy (Figure 1).

\section{Protocol 1}

In this protocol, treatment with G-CSF was started just after administration of DOX to mice. Mice were randomly assigned to receive: (1) DOX alone $(n=11),(2) \mathrm{DOX}+\mathrm{G}-\mathrm{CSF}$ $(n=10)$, (3) saline alone $(n=10)$ or (4) saline + G-CSF $(n=7)$ (Figure 1, Protocol 1a). Two weeks later, all mice were killed with the overdose of pentobarbital following physiological examination. Cardiac specimens were then subjected to histological, immunohistochemical and molecular biological analyses.

In a second set of experiments (Protocol 1b), we evaluated the role played by the extracellular signal-regulated protein kinase (ERK) signaling pathway in mediating the effects of
G-CSF. PD98059 (Cell Signaling), a MEK1-p42/p44 mitogenactivated protein kinase (MAPK)-specific inhibitor, ${ }^{15}$ was administered intraperitoneally at a dose of $0.5 \mathrm{mg} / \mathrm{kg} /$ day for 5 days to mice given saline $(n=6)$ or treated with DOX + G-CSF $(n=6)$. Two weeks later, mice were examined.

In a third set of experiments (Protocol 1c), the role for cyclooxygenase- 2 activation was evaluated in mediating the effects of DOX. NS398, a specific cyclooxygenase-2 inhibitor (Cayman Chemical) ${ }^{16}$ was dissolved in water and given at a dose of $3 \mathrm{mg} / \mathrm{kg} / \mathrm{day}$ for 2 weeks to mice given saline $(n=6)$ or those treated with DOX $(n=6)$ (Figure 1). Mice were examined 2 weeks later.

In a fourth set of experiments (Protocol 1e), we tested the possibility that G-CSF induces bone marrow cell-derived myocardial regeneration. Bone marrow cells were harvested from the femurs and tibias of donor green fluorescent protein (GFP)-transgenic mice with a C57BL/6J background (a gift from Professor Susumu Ikehara, Kansai Medical School, Moriguchi, Japan). ${ }^{17}$ The harvested cells were then transplanted directly into the tibial bone marrow of 8 -week-old male C57BL/6J mice $(n=15)$ that had received whole body irradiation as described previously. ${ }^{18}$ Four weeks later, the 12 surviving chimeric mice were randomly assigned to receive: (1) DOX alone $(n=3)$, (2) DOX + G-CSF $(n=3)$, (3) saline alone $(n=3)$ or (4) saline $+\mathrm{G}-\mathrm{CSF}(n=3)$; the dosages and methods were the same as described in Protocol 1a. On average, $87 \pm 0.88 \%$ of the peripheral blood nucleated cells were positive for GFP in the recipients. Two weeks after DOX injection, all mice were killed and cardiac specimens were subjected to immunohistochemical analyses.

We also used another approach to further clarify the role for bone marrow cell homing in the effect of G-CSF on DOXinduced cardiomyopathy (Protocol 1f). In this fourth set of experiments, AMD3100 (octahydrochloride, Sigma), a specific CXCR4 antagonist, ${ }^{19}$ was administered for 5 days at the dose of $200 \mu \mathrm{g} / \mathrm{kg} /$ day to mice given saline $(n=6)$ or treated with DOX + G-CSF $(n=6)$. Two weeks later, all mice were given a physiological examination.

\section{Protocol 2 (late treatment)}

In this protocol, G-CSF treatment was started 2 weeks after DOX injection. At that time, mice were assigned to a G-CSFtreated group $(n=8)$ or an untreated group $(n=9)$. Before this assignment, echocardiography was carried out to reduce the bias between the groups. Four weeks after DOX injection, mice were examined as described for Protocol 1a (Figure 1).

In a second set of experiments (Protocol 2b), we evaluated animals after a longer follow-up interval (10 weeks): mice received DOX alone or DOX + G-CSF ( $n=13$ each).

\section{Protocol 3 (in vitro experiments)}

Cardiomyocytes were isolated from 1-day-old neonatal C57BL/6 J mice as described previously, ${ }^{20}$ and then plated on laminin-coated dishes or in slide glass chambers and incubated in DMEM (Sigma) supplemented with 10\% FBS 


\section{Protocol 1}

a
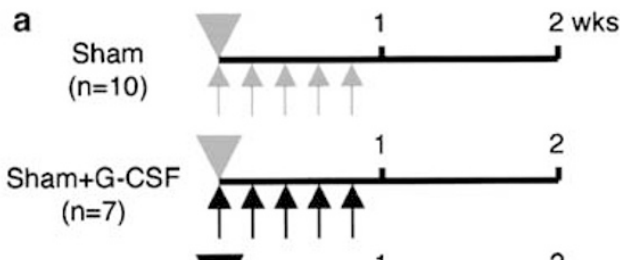

DOX

$(n=11)$

$\mathrm{DOX}+\mathrm{G}-\mathrm{CSF}$

$(n=10)$
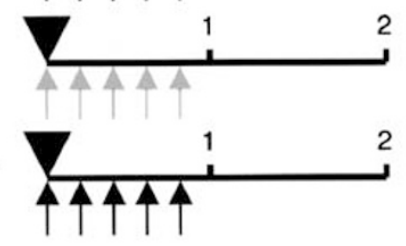

b

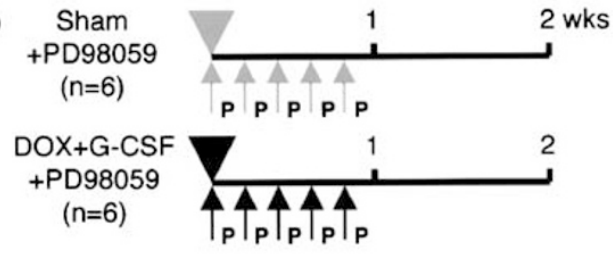

c Sham

+ NS398

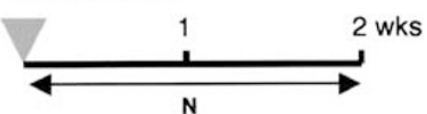

$(n=6)$

DOX

+ NS398

$(n=6)$

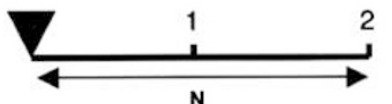

e
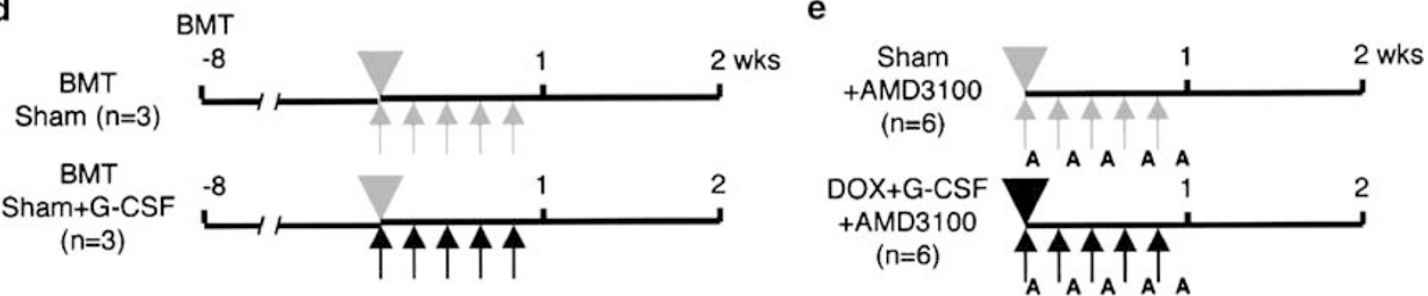
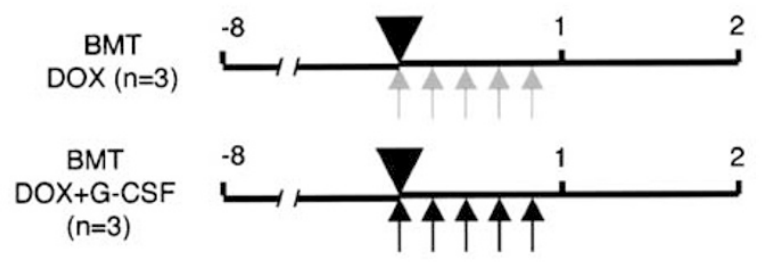

\section{Protocol 2}

a

$\underset{(n=9)}{\operatorname{DOX}}$
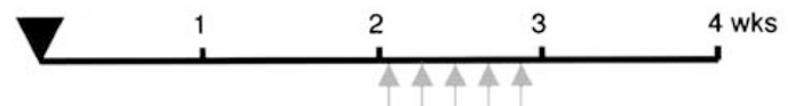

$(n=9)$
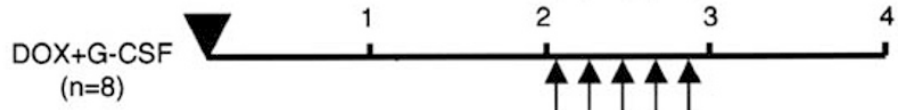

4

b
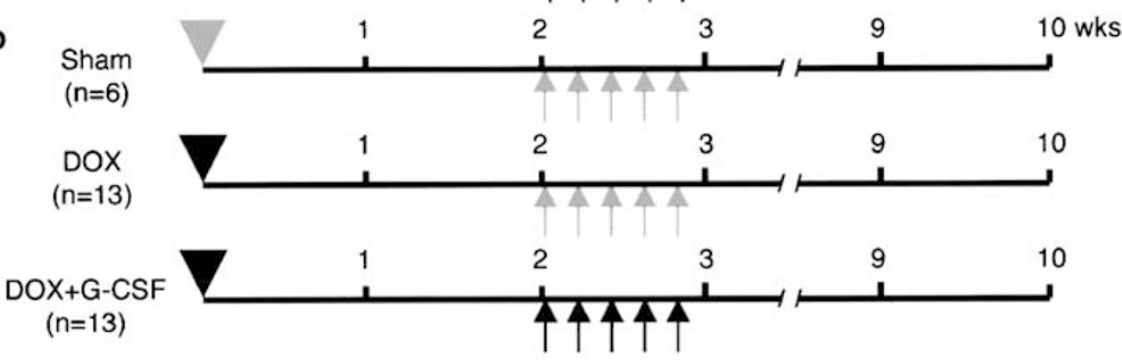

\section{Protocol 3 In vitro experiments}

Figure 1 Experimental protocols. Protocol 1: To examine effect of prophylactic use of G-CSF on DOX-induced cardiomyopathy, G-CSF administration was started immediately after DOX injection. Protocol 2: To examine the therapeutic effect of G-CSF on established DOX-induced cardiomyopathy, G-CSF was started at 2 weeks after DOX injection, when LV dysfunction is already apparent. Mice were examined at 4 or 10 weeks after DOX injection; the latter was to check whether the effect of G-CSF persists for a long interval. Protocol 3: In vitro experiments (see text for details).

(Sigma) for $48 \mathrm{~h}$ at $37^{\circ} \mathrm{C}$. The cells were then treated with DOX at a concentration of $0.1 \mu \mathrm{mol} / 1$ in saline or with the same volume of saline alone. Simultaneously, G-CSF was added to the medium to a final concentration of $0,1,10,50$,
100,1000 or $10000 \mathrm{ng} / \mathrm{ml}$. Survival rate of the in vitro cardiomyocytes was monitored by Trypan blue dye-exclusion at $0,6,12$ and $24 \mathrm{~h}$. Twenty-four hours later, the cells were subjected to morphometric and biochemical analyses. 
Proteins extracted from cardiomyocytes on dishes were used for Western blotting. The cells on slide glass chambers were fixed in $4 \%$ paraformaldehyde, permeabilized with $0.05 \%$ Triton-X and stained with rhodamine-phalloidin and Hoechst 33342 (both from Molecular Probes). Digital images captured using a confocal laser scanning microscope system (LSM510, Zeiss) were used for morphometric analysis using Adobe Photoshop 7.0 (Adobe Systems Inc.).

\section{Physiological Studies}

Echocardiograms were recorded using an echocardiographic system (Aloka) equipped with a 7.5-MHz imaging transducer as described previously. ${ }^{21}$ After cardiac echocardiography, the right carotid artery was cannulated with a micromanometertipped catheter (SPR 671; Millar Instruments) and advanced into the aorta and then into the left ventricle (LV) for recording pressure and $\pm \mathrm{d} P / \mathrm{d} t$.

\section{Histological Analysis}

After echocardiography, each heart was removed and cut into three transverse slices. Of those, the middle slice was fixed with $10 \%$ buffered formalin and embedded in paraffin, after which $4-\mu$ m-thick sections were stained with $\mathrm{H} \& \mathrm{E}$ or Sirius red F3BA ( $0.1 \%$ solution in saturated aqueous picric acid) (Aldrich). Quantitative assessments, including cell size and fibrotic area, were carried out using a multipurpose color image processor (LUZEX F; Nireco, Kyoto, Japan) with 20 randomly chosen high-power fields (HPFs) in each heart.

\section{Immunohistochemical Analysis}

Tissue sections (4- $\mu$ m-thick deparaffinized sections from the middle third of the ventricle or $8-\mu \mathrm{m}$-thick cryosections from the apical third of the ventricle) were incubated with a primary antibody against G-CSF receptor (G-CSFR), Flk-1, myosin heavy chain (MHC) (all from Santa Cruz), GFP (Molecular Probes), $\alpha$-smooth muscle actin ( $\alpha$-SMA) (Sigma) or CD45 (Pharmingen). An ABC kit (DAKO) was then used for immunostaining with diaminobenzidine serving as the chromogen. For immunofluorescence, Alexa Fluor 568 and 488 (Molecular Probes) were the secondary antibodies. Nuclei were counterstained with hematoxylin or Hoechst 33342. The primary antibodies were substituted with the respective control IgG for the control sections. Sections were observed under a light or confocal microscope. For morphometrical analysis of specimens with immunofluorescence, digital images captured using a laser-confocal microscope system (LSM510, Zeiss) were employed for morphometric and quantitative analyses using Adobe Photoshop 7.0 software (Adobe Systems Inc); the number of immunopositive cells (GFP- or CD45-positive cells) was counted using randomly chosen 10 images per tissue section $(4 \mu \mathrm{m})$.

\section{Cell Death Assays}

Previous studies have demonstrated that DOX increases the number of apoptotic cardiomyocytes and induces heart failure. ${ }^{22,23}$ To detect apoptosis, the in situ nick end-labeling (TUNEL) assay was carried out in sections using an ApopTag kit (Intergene) principally according to the supplier's instructions. Mouse mammary tissue served as a positive control. Also, sarcolemmal integrity was assessed by living stain with Evans blue dye. The dye is excluded by cells with normal sarcolemmal permeability but taken up by those with leakier cell membranes including dead cells. ${ }^{24}$ In the animals injected with Evans blue dye 1 day before examination, the hearts were excised and cryosections were examined under a fluorescent microscope. The number of TUNEL- and Evans blue-positive cells was counted in each whole ventricular section. Both TUNEL assay and Evans blue staining were performed in the mice at $1,3,7$ and 14 days ( $n=3$ each) after treatment with saline or DOX.

\section{Electron Microscopy}

Cardiac specimens were immersion-fixed overnight in phosphate-buffered $2.5 \%$ glutaraldehyde ( $\mathrm{pH} 7.4$ ), postfixed for $1 \mathrm{~h}$ with $1 \%$ osmium tetroxide, dehydrated through a graded ethanol series, and embedded in Epon medium. Ultrathin sections were stained with uranyl acetate and lead citrate and observed in an electron microscope ( $\mathrm{H} 700$, Hitachi, Tokyo, Japan).

\section{Western Analysis}

Heart tissue lysates were used for Western analysis. Proteins were separated and transferred to membranes using standard protocols, after which they were probed using antibodies against G-CSFR, GATA-4, MHC, troponin I (all from Santa Cruz), desmin (Sigma), COX-2 (Santa Cruz) or transforming growth factor- $\beta 1$ (TGF- $\beta 1$, Promega). Activation of Akt, signal transducer and activator of transcription-3 (STAT3) and ERK, downstream mediators of G-CSFR signaling, was assessed using antibodies against the phosphorylated forms of Akt (p-Akt), p-STAT3 and p-ERK (all from Cell Signaling). Procaspase- 3 and the activated form of caspase- 3 were evaluated using anti-caspase- 3 antibody (Santa Cruz). Three to five hearts from each group were subjected to the blotting. The blots were visualized by means of chemiluminescence (ECL, Amersham), and the signals were quantified by densitometry. $\alpha$-tubulin (analyzed by antibody from Santa Cruz) served as the loading control.

\section{ELISA}

Levels of tumor necrosis factor- $\alpha$ (TNF- $\alpha$ ) in the myocardium were assayed using an ELISA (R\&D Systems). Three hearts from each group were used for this assay.

\section{Statistical Analysis}

Value are shown as means \pm s.e.m. The significance of differences between groups was evaluated using $t$-tests or oneway ANOVA followed by Newman-Keul's multiple comparison test. Values of $P<0.05$ were considered significant. 


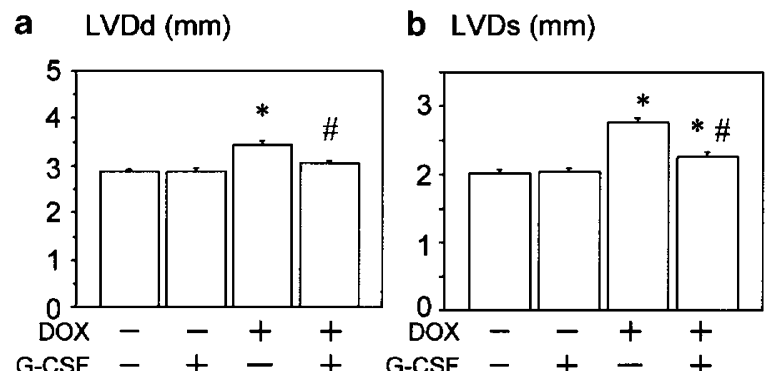

C $\% \mathrm{FS}(\%)$
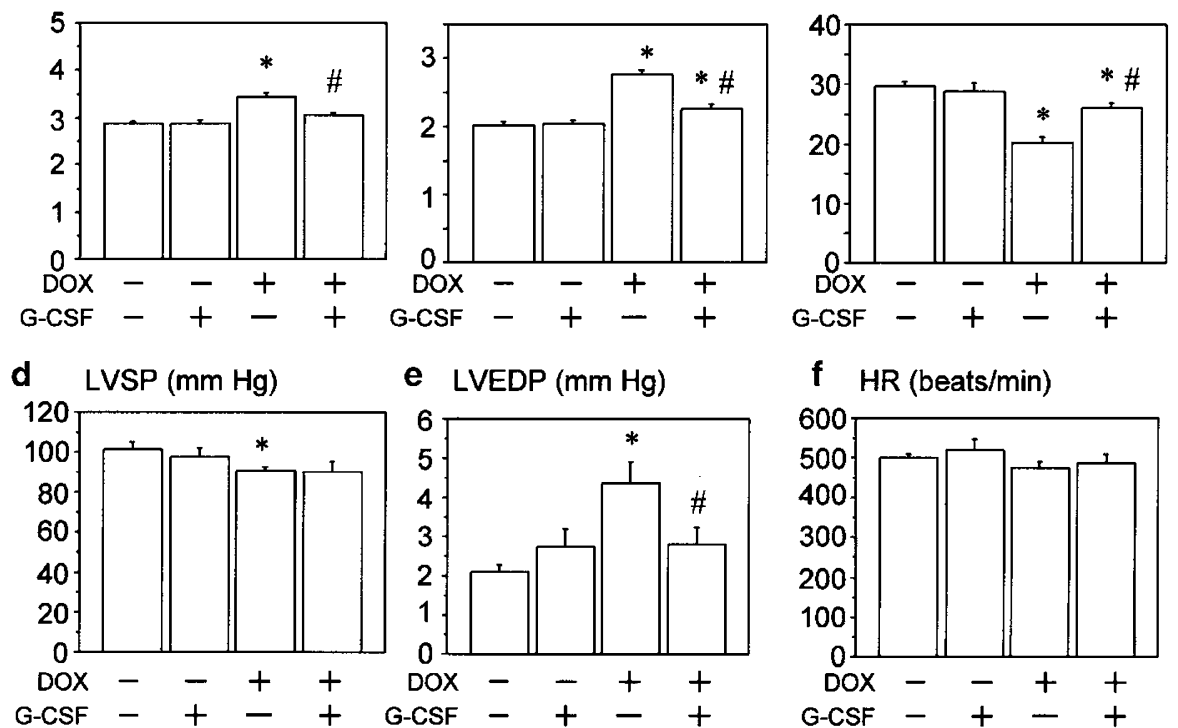

f $H R$ (beats/min)

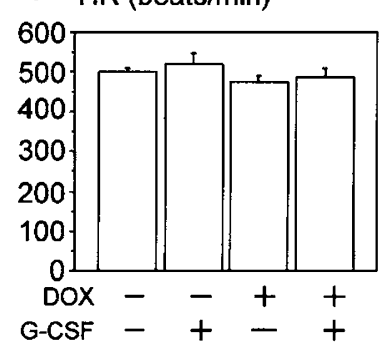

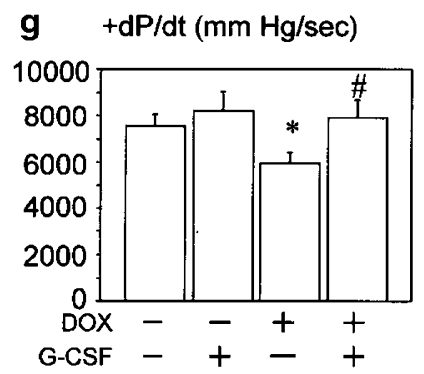

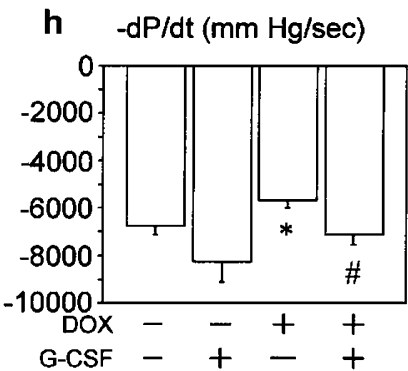

Figure 2 Effects of G-CSF on LV geometry and function assessed 2 weeks after DOX injection (Protocol 1a). The indicated parameters were measured using echocardiography and cardiac catheterization. Bars are means \pm s.e.m.; ${ }^{*}<<0.05$ vs the untreated sham group (DOX $(-)$ and $\left.\mathrm{G}-\mathrm{CSF}(-)\right)$; ${ }^{\#} P<0.05$ vs the untreated DOX group (DOX (+) and G-CSF (-)).

\section{RESULTS}

\section{Effects of Prophylactic Use of G-CSF on DOX-Induced Cardiomyopathy}

In Protocol 1a (Figure 1), G-CSF was administered at the dose of $100 \mu \mathrm{g} / \mathrm{kg} /$ day for 5 days, beginning immediately after a single injection of DOX $(15 \mathrm{mg} / \mathrm{kg})$ to mice. Two weeks after DOX administration, all of the mice in the untreated $(n=11)$ and G-CSF-treated $(n=10)$ groups and in the sham groups (untreated, $n=10$ and G-CSF-treated, $n=7$ ) were alive. Granulocyte count on the day after the last treatment were significantly greater in the G-CSF-treated groups (sham, 3713 $\pm 511 / \mu \mathrm{l}$; DOX, 3218 $\pm 644 / \mu \mathrm{l})$ than the salinetreated (sham, $1322 \pm 205 / \mu \mathrm{l}(P<0.05) ;$ DOX, $471 \pm 89 / \mu \mathrm{l}$ $(P<0.05))$ groups, but had returned to control levels by the time of sacrifice (2 weeks later).

Echocardiography and cardiac catheterization showed that, as compared with sham animals, mice receiving DOX alone had significant deterioration of LV functionality characterized by enlargement of the LV cavity and increased LV diameters (LVDd, $3.5 \pm 0.085$ vs $2.8 \pm 0.065 \mathrm{~mm}$ in sham $(P<0.05))$ and end-diastolic pressures (LVEDP, $4.3 \pm 0.67 v s$
$1.9 \pm 0.19 \mathrm{~mm} \mathrm{Hg}$ in sham $(P<0.05))$ and decreased LV fractional shortening (LVFS, $18 \pm 0.48$ vs $32 \pm 1.2 \%$ in sham $(P<0.05))$ and $\pm \mathrm{d} P / \mathrm{d} t(+\mathrm{d} P / \mathrm{d} t, \quad 5569 \pm 218$ vs $7916 \pm$ $393 \mathrm{~mm} \mathrm{Hg} / \mathrm{s}$ in sham $(P<0.05) ;-\mathrm{d} P / \mathrm{d} t,-5764 \pm 309$ vs $-7391 \pm 250 \mathrm{~mm} \mathrm{Hg} / \mathrm{s}$ in sham $(P<0.05)$ ) (Figure 2). Treatment with G-CSF significantly mitigated the DOXinduced impairment of cardiac function (LVDd, 3.1 $0.042 \mathrm{~mm} ; \quad$ LVEDP, $\quad 2.4 \pm 0.38 \mathrm{~mm} \mathrm{Hg} ; \quad$ LVFS, $27 \pm 0.79 \%$; $+\mathrm{d} P / \mathrm{d} t, 7852 \pm 390 \mathrm{~mm} \mathrm{Hg} / \mathrm{sec} ;-\mathrm{d} P / \mathrm{d} t,-7108 \pm 419 \mathrm{~mm} \mathrm{Hg} / \mathrm{s}$ (all $P<0.05$ compared with the saline-treated group)). Administration of G-CSF had no effect on cardiac function of the sham mice.

We observed no significant difference in the heart-to-body weight ratios among the groups in Protocol 1a (sham, $3.71 \pm 0.05 \mathrm{mg} / \mathrm{g}$; G-CSF alone, $3.79 \pm 0.15 \mathrm{mg} / \mathrm{g}$; DOX alone, $3.86 \pm 0.10 \mathrm{mg} / \mathrm{g} ; \quad$ DOX plus G-CSF, $3.83 \pm 0.12 \mathrm{mg} / \mathrm{g}$ $(P=\mathrm{NS}))$. On the other hand, examination of transverse sections of hearts stained with H\&E (Figure 3a) revealed that the transverse diameters of cardiomyocytes from the group receiving DOX alone were significantly smaller than in the sham group $(13.7 \pm 0.1$ vs $14.6 \pm 0.1 \mu \mathrm{m}(P<0.05))$, and that 
a

HE
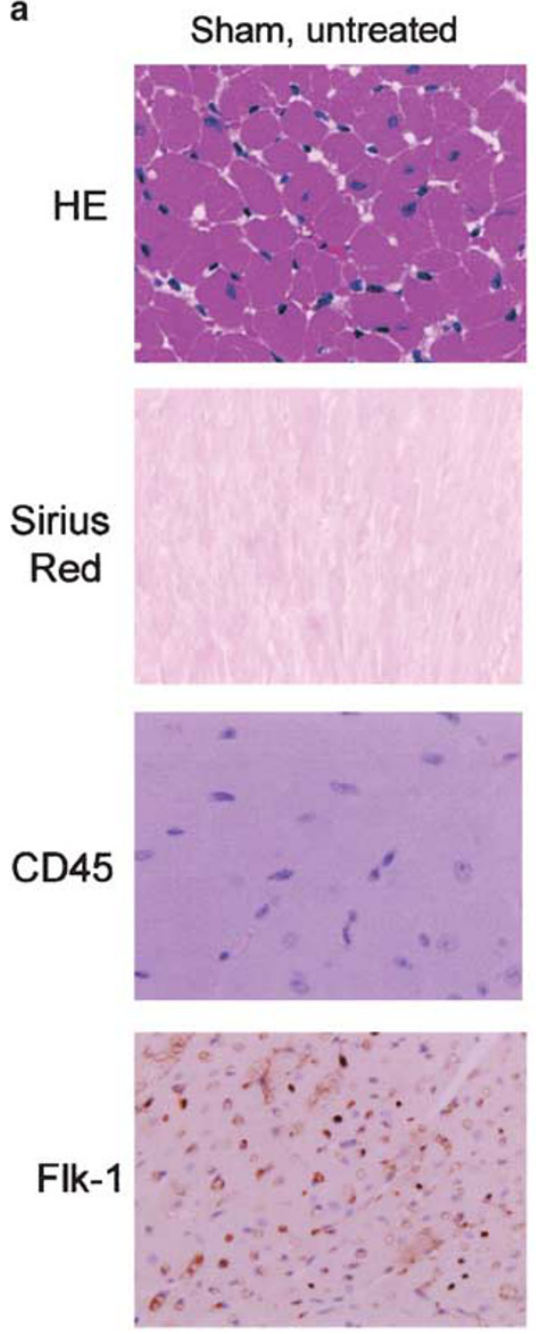

DOX, untreated
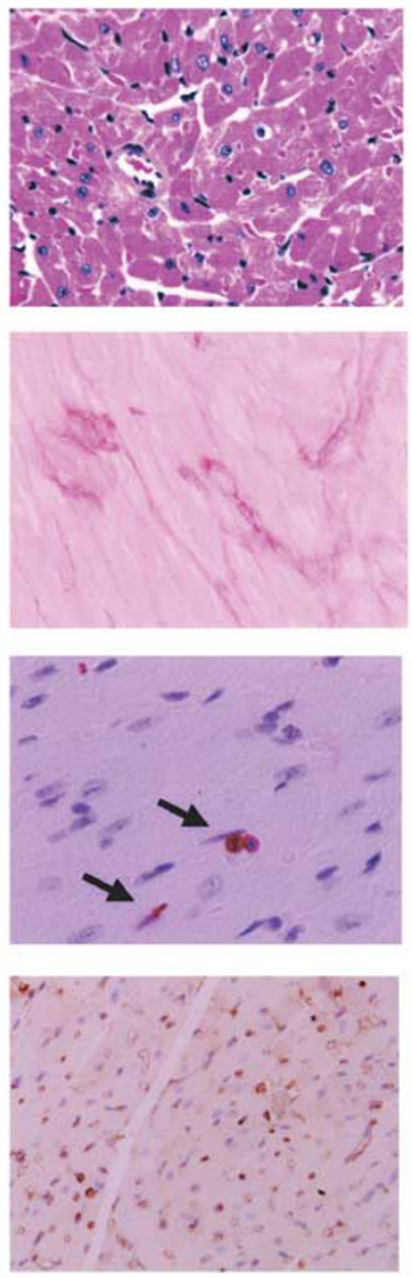

DOX, G-CSF-treated
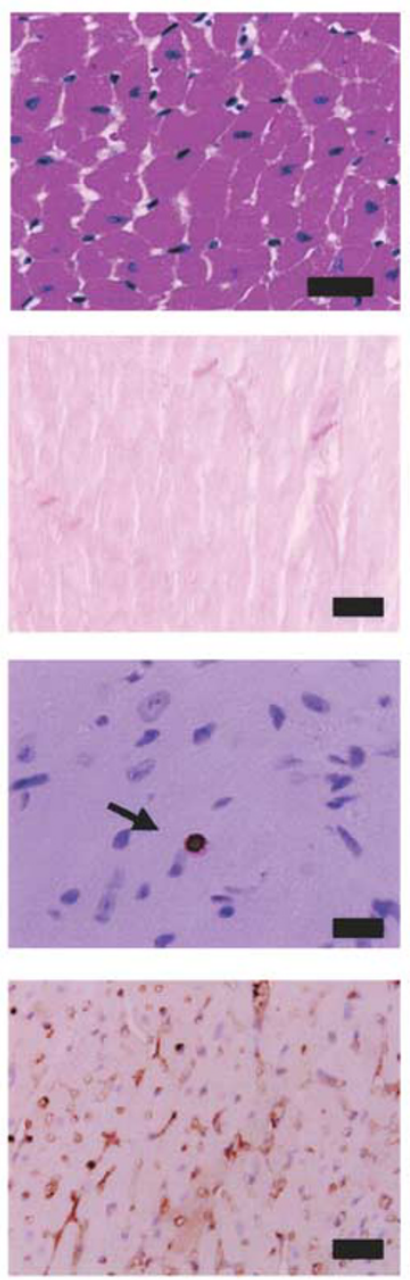

b
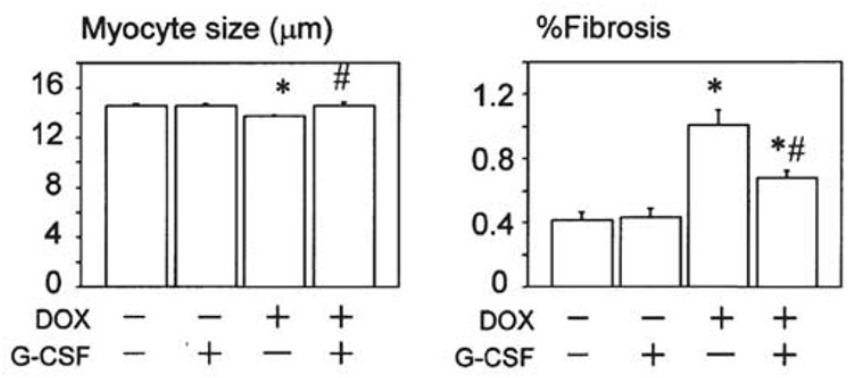

CD45-positive cells (/HPF)

Flk-1-positive cells (/HPF)
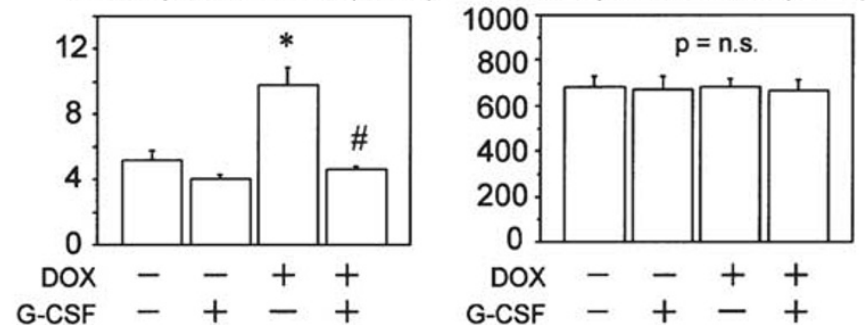

Figure 3 Effects of G-CSF on cardiac histology in mice 2 weeks after DOX injection (Protocol 1a). (a) Photomicrographs of histological (H\&E and Sirius redstained) and immunohistochemical (CD45 and Flk-1) preparations of heart specimens from the indicated groups. Arrows point to immunopositive cells; scale bars, $20 \mu \mathrm{m}$. (b) Graphs showing morphometric data. Bars are means \pm s.e.m.; ${ }^{*} P<0.05$ vs the untreated sham group (DOX (-) and G-CSF $\left.(-)\right) ;{ }^{\#} P<0.05$ vs the untreated DOX group (DOX (+) and G-CSF (-)). 
G-CSF exerted a significant protective effect against such DOX-induced cardiomyocyte atrophy (transverse diameter, $14.5 \pm 0.3 \mu \mathrm{m} \quad(P<0.05)$ ) (Figure $3 \mathrm{~b})$. Similarly, when we assessed myocardial fibrosis using Sirius red-stained sections, we found significantly more fibrosis in the group receiving DOX alone than in the sham group $(1.01 \pm 0.10$ vs $0.42 \pm$ $0.05 \% \quad(P<0.05))$ or the group receiving DOX + G-CSF $(0.68 \pm 0.04 \%(P<0.05))$ (Figure 3$)$. In addition, immunohistochemical analysis revealed that DOX initiated significant infiltration of the myocardium by CD45-positive leukocytes $(9.8 \pm 1.1$ vs $5.2 \pm 0.6$ cells/HPF in the sham group $(P<0.05))$, and again G-CSF attenuated DOX's effect, reducing the leukocyte infiltration to $4.6 \pm 0.2$ cells/HPF $(P<0.05)$. There was no significant difference in the number of Flk-1-positive endothelial cells among the groups (Figure 3).

Degenerative changes within cardiomyocytes caused by DOX were clearly evident on electron micrographs, which confirmed previously described findings in DOX cardiomyopathy. ${ }^{6,25}$ These changes were characterized by myofibrilar derangement and disruption and by increases in the volumes of subcellular organelles (Figure 4). These degenerative changes were significantly attenuated by treatment with G-CSF.

TUNEL-positive cells were observed among both cardiomyocytes and noncardiomyocytes from all groups, and we found no significant difference in the incidence of TUNEL positivity between mice that received DOX $(0.13 \pm 0.048 \%$ in myocytes; $0.021 \pm 0.011 \%$ in nonmyocytes) and those that did not $(0.084 \pm 0.056 \%$ in myocytes; $0.035 \pm 0.021 \%$ in nonmyocytes (both $P=\mathrm{NS}$ )); G-CSF had no significant effect on the incidence of TUNEL positivity $(0.093 \pm 0.064 \%$ in myocytes; $0.036 \pm 0.017 \%$ in nonmyocytes (both $P=\mathrm{NS}$ )) (Figure 4). Consistent with those findings, we detected no activated caspase- 3 in any of the groups. And although as mentioned above electron microscopy revealed degenerative changes within cardiomyocytes from DOX-treated mice (Figure 4), typical apoptotic ultrastructure was never seen. We also found no Evans blue-positive cardiomyocytes in any mice that received DOX or not at any time points examined (Figure 4). Collectively, neither apoptosis nor death of cardiac cells seems to be critically involved in the present model of DOX-induced cardiomyopathy whereas DOX-induced degenerative changes were apparent in the cardiomyocytes.
No adverse reactions to G-CSF, including splenic rupture, thromboembolism, interstitial pneumonia and atherosclerosis, were detected.

\section{Mechanisms for Beneficial Effect of G-CSF on DOX-Induced Cardiomyopathy}

GATA-4 is a key transcriptional factor regulating expression of sarcomeric proteins in the heart. ${ }^{26,27}$ We found myocardial levels of GATA-4 to be significantly reduced by DOX (Figure 3 ), confirming earlier reports. ${ }^{22,23}$ We also found that this reduction was partially but significantly restored by G-CSF treatment (Figure 5). Likewise, levels of three sarcomeric proteins, MHC, troponin I and desmin, were significantly reduced by DOX, and this inhibitory effect was also significantly reversed by G-CSF. DOX had no significant effect on myocardial expression of TNF- $\alpha$ or TGF- $\beta 1$; however, cyclooxygenase- 2 expression was markedly upregulated by DOX, and that effect was largely reversed by G-CSF (Figure 6). Western analysis revealed that expression of G-CSF receptor (G-CSFR) was significantly diminished in DOXtreated hearts, but was greatly enhanced by G-CSF treatment (Figure 7a). Consistent with this finding, immunohistochemical analysis showed G-CSFR to be expressed on cardiomyocytes and to be more strongly expressed in G-CSFtreated hearts (Figure 7a).

ERK/MAPK, receptor-associated Janus family tyrosine kinase (Jak)/STAT and/or phosphatidylinositol 3-kinase (PI3K)/Akt are known to serve as downstream mediators of G-CSFR signaling in hematopoietic cell lines ${ }^{28,29}$ and myocardium. ${ }^{14}$ We found that neither DOX-induced cardiomyopathy nor the effects of G-CSF were related to activation (phosphorylation) of Akt or STAT3 (Figure 7b). On the other hand, ERK phosphorylation, and thus its activation, was markedly inhibited by DOX, and that effect was significantly attenuated by G-CSF (Figure 7b). To further confirm the critical role played by ERK in mediating the cardioprotective effects of G-CSF, we next tested the effect of inhibiting ERK activation using the MEK1-p42/p44 MAPK-specific inhibitor PD98059 (Protocol 1b). When administered to mice along with DOX + G-CSF, PD98059 suppressed ERK activation and GATA-4 expression to the same level seen in untreated DOXinduced cardiomyopathy (Figure 8a). Moreover, PD98059 completely eliminated the G-CSF-mediated improvement in cardiac function and histology (Figure $8 \mathrm{~b}$ and $\mathrm{c}$ ), suggesting that the ERK pathway is critically involved in the protective

Figure 4 DOX-induced degeneration, apoptosis and cell death in the heart. (a) Electron micrographs of cardiomyocytes showing marked DOX-induced degenerative changes (left panel) that were significantly attenuated by G-CSF (right panel). Bars, $1 \mu \mathrm{m}$. (b) TUNEL assay. Photomicrographs showing TUNELpositive cells in the myocardium: left panel, a TUNEL-positive cardiomyocyte; right panel, a TUNEL-positive nonmyocyte. Arrows point to TUNEL-positive cells. Bars, $20 \mu \mathrm{m}$. Graphs show the TUNEL assay-based apoptotic index among cardiomyocytes (left) and nonmyocytes (right) at 14 days after the treatments. (c) The graph shows the apoptotic index of cardiomyocytes at 1, 3 and 7 days after DOX treatment. (d) Western analysis of caspase-3 expression. The active (cleaved) form of caspase-3 was not detectable in hearts from any of the groups. (e) Evans blue staining of myocardium from the heart treated with DOX. Fluorescent microphotograph shows Evans blue was exclusively confined in vessel lumens, but never in myocardial parenchyma, in hearts from any group. Bar, $20 \mu \mathrm{m}$. (f) Survival rate of cultured cardiomyocytes treated with saline (S) or DOX (D) and monitored with Trypan blue dye exclusion for the indicated intervals. 
a

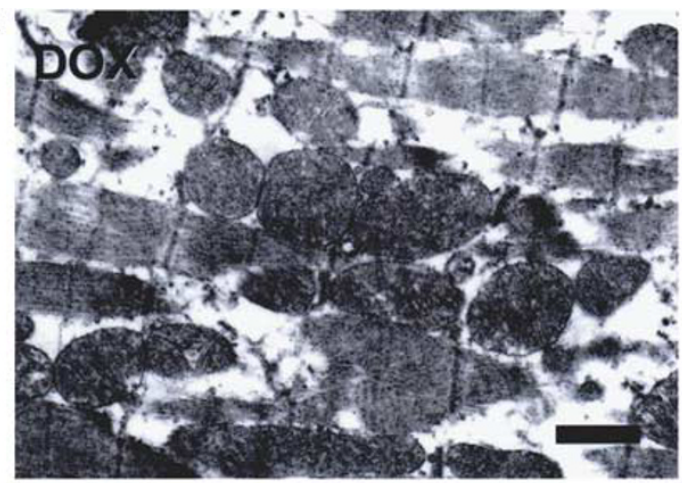

b

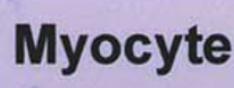

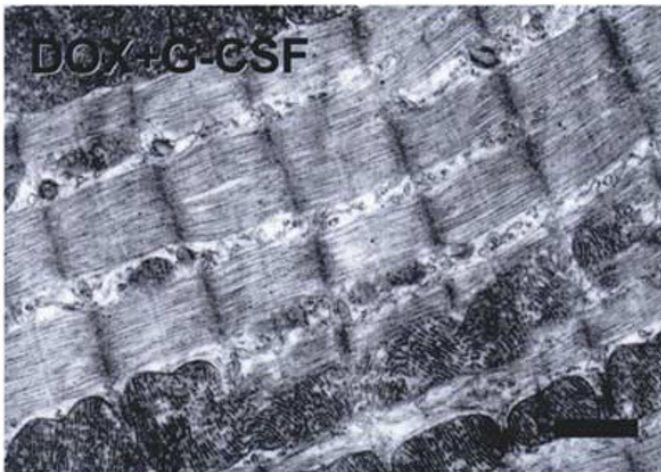
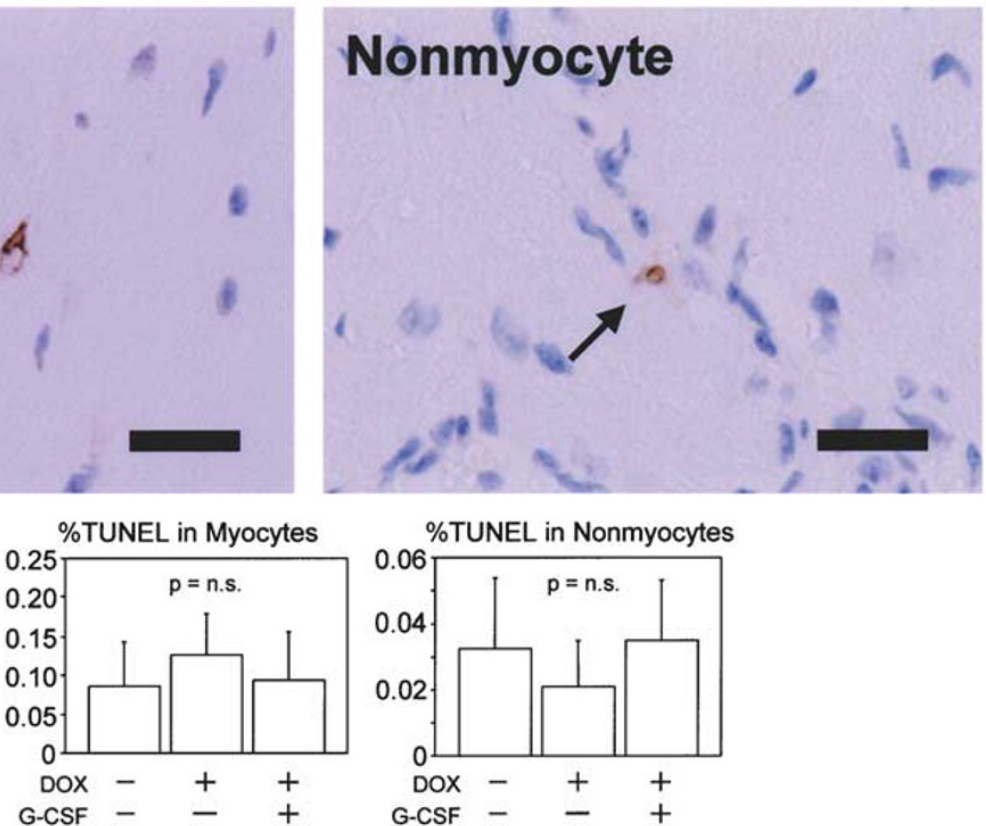

c Progression of \%TUNEL ${ }^{+}$ Myocytes after DOX Treatment

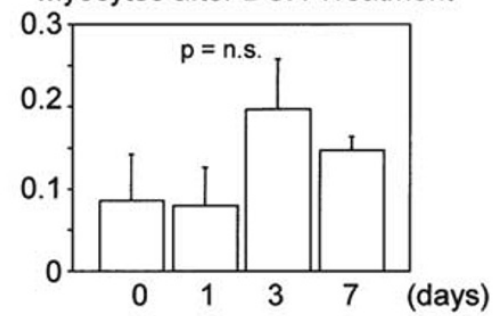

e

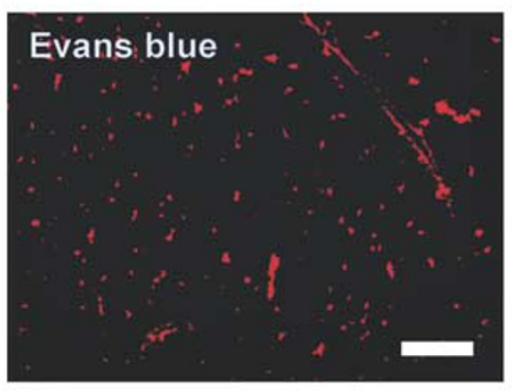

d

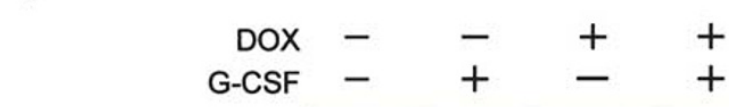

Procaspase-3

Cleaved caspase- 3

Tubulin

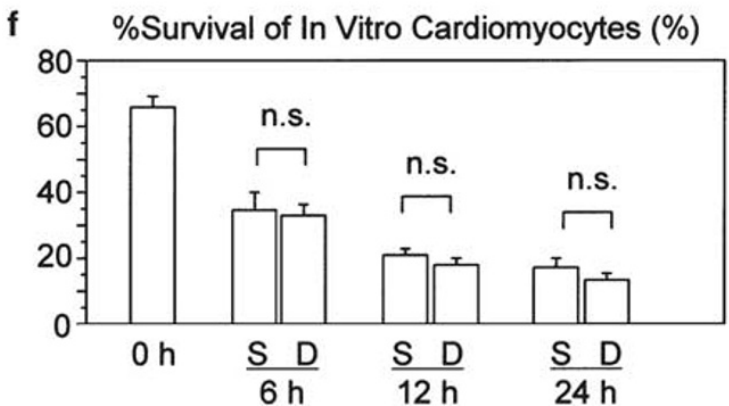


a
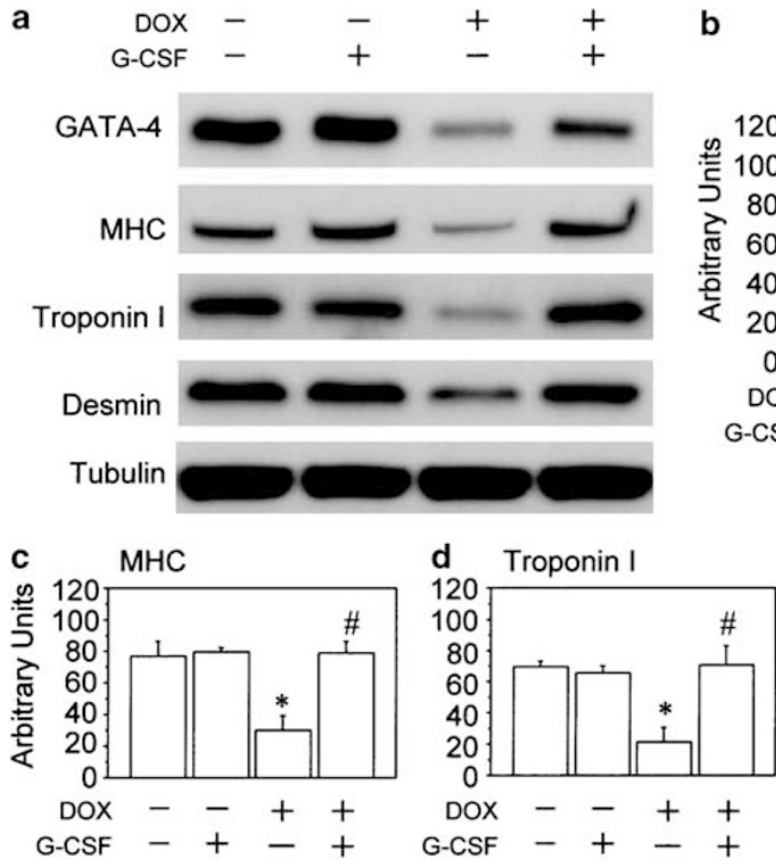

b

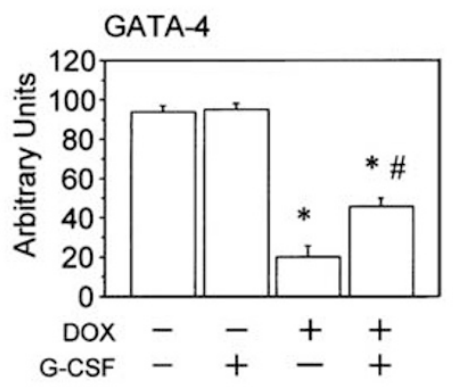

Figure 5 Western analysis of the effects of G-CSF on myocardial expression of GATA-4 and three sarcomeric proteins: MHC, troponin I and desmin Bars are means \pm s.e.m.; ${ }^{*} P<0.05$ vs the untreated sham group (DOX (-) and G-CSF $(-)$ ); ${ }^{\#} P<0.05$ vs the untreated DOX group (DOX $(+)$ and G-CSF (-)). effect exerted by G-CSF against DOX-induced cardiomyopathy.

To evaluate the role for COX-2 activation in mediating the effect of DOX on the heart of the present model, we checked the effect of inhibiting cyclooxygenase- 2 activation using the specific cyclooxygenase-2 inhibitor NS398 (Protocol 1c). This experiment revealed that NS398 could not beneficially affect cardiac function in DOX-induced cardiomyopathy although it partially suppressed myocardial inflammation and fibrosis (Figure $8 \mathrm{~b}$ and c). In addition, NS398 was confirmed not to reverse DOX-induced ERK inactivation and GATA-4 reduction (Figure 6d). On the other hand, ERK inhibition by PD 98059 did not restore the downregulated COX-2 by G-CSF (Figure 8e).

In an experiment using chimeric mice transplanted with GFP transgenic mouse-derived bone marrow cells (Protocol 1e), we observed no significant effect of G-CSF on mobilization and cardiac homing of bone marrow cells (Figure 9). That is, our examination of cellular immunofluorescence revealed no cardiomyocytes, endothelial cells or $\alpha$-SMApositive vascular smooth muscle cells originating from GFPpositive cells; some CD45-positive cells expressing GFP were detected in the myocardium, however (Figure 9). AMD3100 is a specific CXCR4 antagonist that prevents homing of CXCR4 ${ }^{+}$cells. When administered to mice receiving DOX + G-CSF (Protocol 1f), AMD3100 had no effect on the G-CSFmediated improvement in cardiac function (Figure $8 \mathrm{~b}$ ).

\section{Effects of G-CSF on Established DOX-Induced Cardiomyopathy}

Using Protocol 2 (Figure 1), we next examined the therapeutic efficacy of G-CSF on established LV dysfunction re- sulting from DOX-induced cardiomyopathy (late treatment). In this experiment, G-CSF treatment was started 2 weeks after DOX injection, at which time LV dysfunction was readily apparent; animals were then examined 2 weeks later. Under these conditions, we found that G-CSF still had significant beneficial, and thus therapeutic, effects on DOXinduced cardiomyopathy (Table 1).

We next evaluated longer effect of G-CSF on DOX-induced cardiomyopathy (Figure 1, Protocol 2b). The survival rate of mice in Protocol 2b was similar between the salineand G-CSF-treated groups (77 vs 77\%). However, as shown in Table 2, we noted that a significant cardiac protection by G-CSF persisted for a long interval because the cardiac function was significantly better even after the follow-up for 10 weeks.

\section{In Vitro Effects of G-CSF on Cardiac Cells (Protocol 3)}

Cultured cardiomyocytes incubated in the presence of DOX $(0.1 \mu \mathrm{mol} / \mathrm{l})$ showed significant atrophy/degeneration. G-CSF treatment significantly diminished the DOX-induced cardiomyocyte atrophy (Figure 10a). The effect of G-CSF on cardiomyocytes was dose-dependent and reached a maximum at a concentration of $100 \mathrm{ng} / \mathrm{ml}$. Western analysis revealed that DOX significantly reduced expression of GATA4 and MHC in cultured cardiomyocytes, both of which were restored by G-CSF treatment (Figure 10b). In addition, DOX significantly increased expression of cyclooxygenase-2, and that effect, too, was significantly reversed by G-CSF (Figure 10c). Thus, G-CSF appears to act directly on cardiomyocytes to exert its anti-atrophic and anti-inflammatory effects. DOX at the present concentration did not affect the time course of the survival rate of cardiomyocytes (Figure 4). 
a

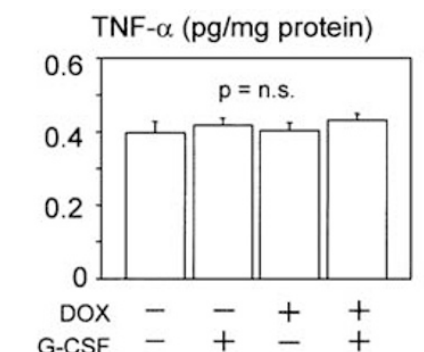

b

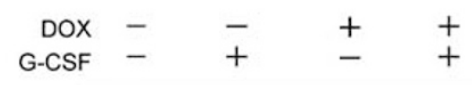

TGF- $\beta 1$

COX-2

Tubulin

TGF- $\beta 1$

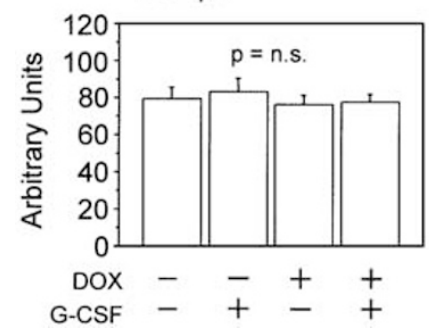

Figure 6 (a) Myocardial levels of TNF- $\alpha$ (pg/mg protein) determined by ELISA. (b) Western analysis of the effects of DOX and G-CSF on myocardial expression of TGF- $\beta 1$ and COX-2. Bars are means \pm s.e.m.; ${ }^{*} P<0.05$ vs the untreated sham group (DOX (-) and G-CSF (-)); ${ }^{\#} P<0.05$ vs the untreated DOX group (DOX $(+)$ and G-CSF $(-))$.

\section{DISCUSSION}

\section{G-CSF Exerts a Protective Effect Against DOX-Induced Cardiomyopathy}

The present study provides the evidence of the beneficial effects of G-CSF on LV dysfunction caused by DOX-induced cardiomyopathy, a nonischemic cardiomyopathy. The principle pathologic findings were that G-CSF prevented DOXinduced atrophic degeneration of cardiomyocytes and cardiac fibrosis. The mechanism of action of G-CSF in this model differs from that seen in cases of myocardial infarction, where G-CSF reportedly enhances the survival of ischemic cardiomyocytes. ${ }^{13,14}$ What's more, G-CSF also exerted a therapeutic effect on DOX-induced cardiac dysfunction and histopathologic changes after the cardiomyopathy had become established.

\section{Mechanisms Underlying the Cardioprotective Effects of G-CSF}

Our findings suggest that several factors contribute to the cardioprotective effects of G-CSF on DOX-induced cardiomyopathy. The first is that G-CSF mitigates the evoked atrophic degeneration of cardiomyocytes. Sarcomeric proteins, including MHC, troponin I and desmin, are important for the structural integrity and function of cardiomyocytes, and their myocardial expression is reportedly downregulated by DOX, ${ }^{5}$ an effect we confirmed in the present study. Our new finding is that G-CSF significantly restores the expression of sarcomeric proteins in the presence of DOX. We suggest that GATA-4 is involved in this anti-atrophic effect, as GATA-4 is a key regulator of heart development that also is known to regulate myocardial expression of MHC and troponin $\mathrm{I}^{26,27}$ and was recently shown to be depleted in DOXinduced cardiotoxicity. ${ }^{22,23}$ Our results not only confirmed that earlier finding, but also demonstrated that G-CSF restores GATA-4 expression in the presence of DOX.

In hematopoietic and cardiac cells, G-CSFR signaling can activate ERK/MAPK, PI3K/Akt and Jak/STAT3 signaling pathways. ${ }^{14,28,29}$ Our findings suggest that altered signaling via ERK, but not Akt or STAT3, is involved in DOX-induced cardiomyopathy, which is consistent with a very recent study showing that ERK activation is significantly diminished during the chronic stage of DOX-induced cardiomyopathy (3 weeks after DOX injection). ${ }^{30}$ Given that another study using isolated rat heart subjected to excessive LV wall stress (induced by balloon inflation) showed an involvement of MAPK (p38 and ERKs) in the activation of GATA-4 binding to DNA, ${ }^{31}$ we suggest that G-CSF exerts its cardioprotective effects via the ERK/MAPK signaling pathway, which was otherwise inhibited by DOX. We recently showed that G-CSF improved cardiac function of the mouse heart with a large old MI. ${ }^{21}$ In that model, G-CSF-induced molecular signaling downstream of G-CSFR differed substantially from that seen in the present model despite the animal species used was same. Stat and Akt activation was augmented in the mouse heart with old MI, which was furthermore strengthened by G-CSF, whereas ERK activation was not influenced by G-CSF. Thus, G-CSF's downstream signal via G-CSFR apparently varies in different models of heart failure.

We observed that DOX stimulates both myocardial infiltration by inflammatory cells and the development myocardial fibrosis, and that G-CSF prevents these pathological processes. Similar anti-inflammatory properties of G-CSF were observed previously in the central nervous system and in monocytes. ${ }^{32,33}$ In the heart, induction of a powerful inflammatory mediator is reportedly associated with heart failure. ${ }^{34}$ Although earlier studies reported TNF- $\alpha$ and TGF$\beta 1$ to be potent stimulators of inflammation and fibrosis in the failing heart, ${ }^{35,36}$ their involvement in DOX-induced cardiomyopathy was challenged in a recent report. ${ }^{37}$ Consistent with the latter, we found no significant DOX- or GCSF-induced changes in the expression of TNF- $\alpha$ and TGF$\beta 1$. On the other hand, it is known that DOX induces myocardial expression cyclooxygenase- $2,{ }^{38}$ which occupies a central position in the biosynthesis of proinflammatory prostaglandin $\mathrm{E} 2$, prostacyclin and thromboxane $\mathrm{A}_{2}$, and that inhibition of cyclooxygenase-2 improves cardiac function in DOX-induced cardiomyopathy. ${ }^{39}$ Actually, we noted that DOX strongly induced myocardial cyclooxygenase-2 
a
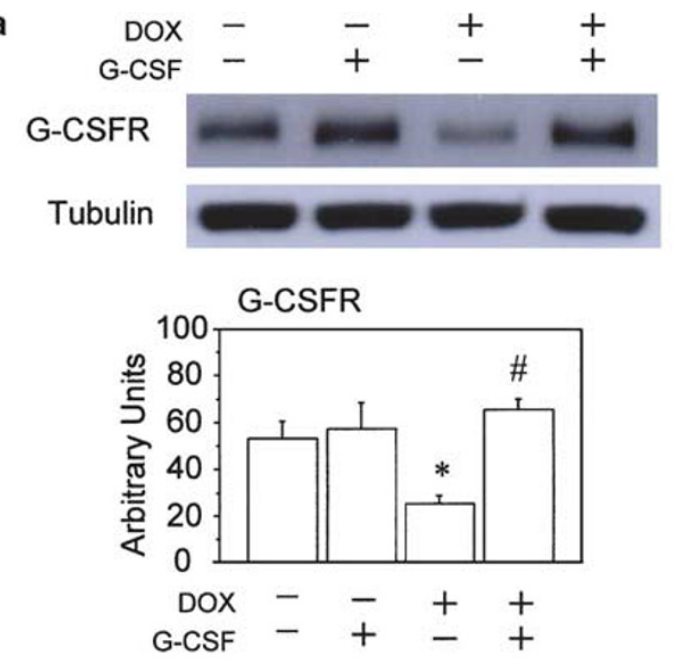

DOX

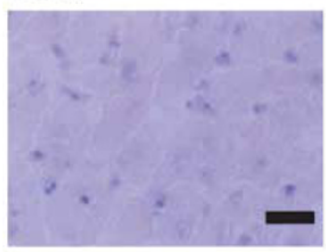

\section{$\mathrm{DOX}+\mathrm{G}-\mathrm{CSF}$}

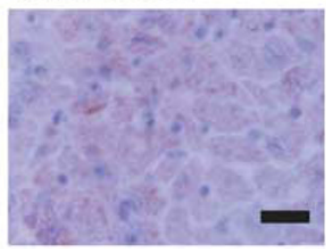

Figure 7 (a) Western (left) and immunohistochemical (right) analyses of the effect of G-CSF on G-CSFR expression in heart. Bars, $20 \mu \mathrm{m}$. (b) Western analysis of the downstream targets of G-CSFR signaling. The degree of activation of the PI3K/Akt, Jak/STAT and ERK/MAPK signaling pathways was assessed by determining the levels of the phosphorylated forms of Akt, STAT3 and ERK (p-Akt, p-STAT3 and p-ERK, respectively). Bars are means \pm s.e.m.; ${ }^{*} P<0.05$ vs the untreated sham group (DOX (-) and G-CSF (-)); ${ }^{\#} P<0.05$ vs the untreated DOX group (DOX $(+)$ and G-CSF (-)).

b

$$
\begin{array}{rrrrr}
\mathrm{DOX} & - & - & + & + \\
\mathrm{G}-\mathrm{CSF} & - & + & - & +
\end{array}
$$
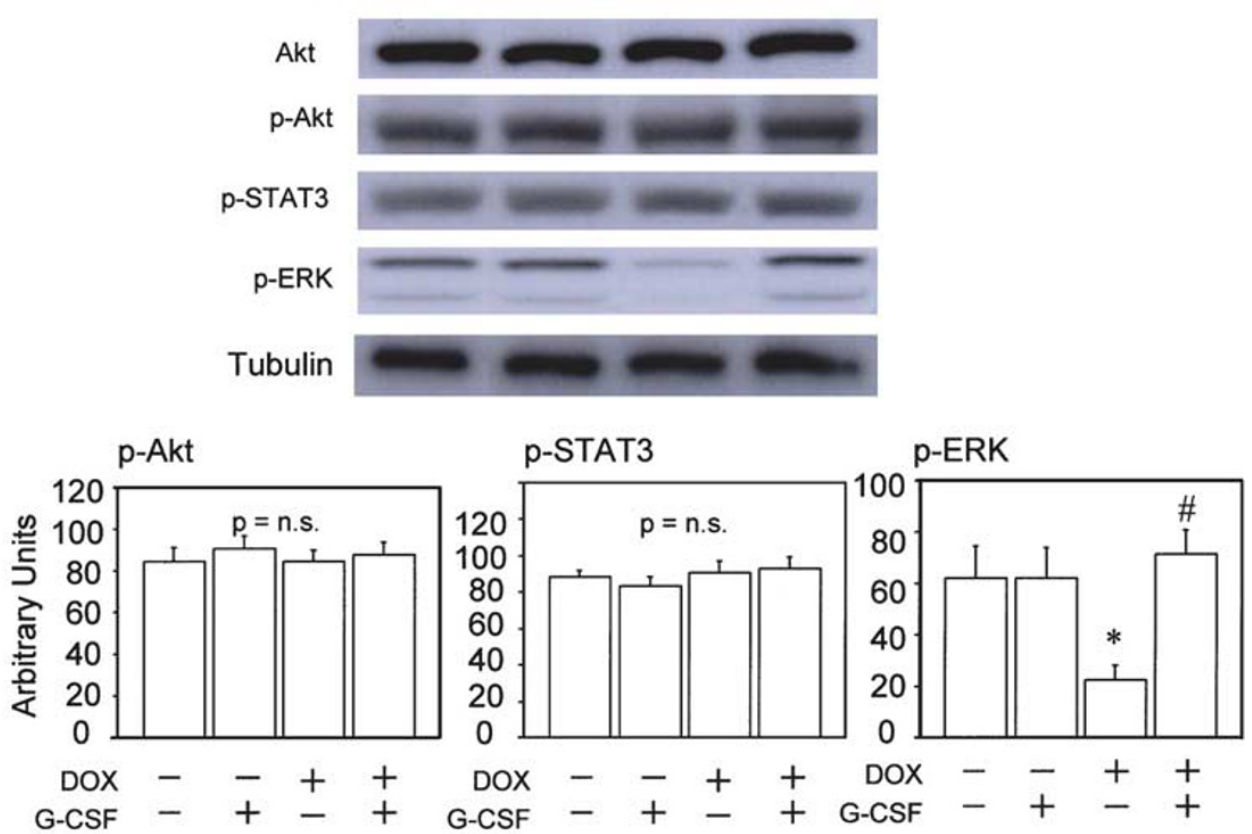

expression in the present study and that this effect was largely inhibited by G-CSF. However, we also found that inhibition of cyclooxygenase- 2 by a specific cyclooxygenase-2 inhibitor could not mimic the effect of G-CSF on DOX-induced cardiomyopathy: no improvement in cardiac function or no restoration of cardiomyocyte size, though with partial reduction in myocardial inflammation and fibrosis. PD98059 and NS398 did not affect, respectively, cyclooxygenase-2 expression and ERK activation. Collectively, these findings indicate that myocardial fibrosis and inflammation induced by DOX may be regulated by cyclooxygenase- 2 upregulation although these phenotypes are unlikely to profoundly affect the cardiac function and that anti-atrophic/degenerative action of G-CSF on cardiomyocytes that is accompanied by ERK-mediated GATA-4 expression is the most important mechanism of the beneficial effects of G-CSF against cardiac dysfunction in DOX-induced cardiomyopathy.

Our immunohistochemical and Western blot analyses showed strong myocardial expression of G-CSFR, which confirms earlier reports. ${ }^{14,21}$ We also found that DOX significantly downregulated myocardial G-CSFR expression and that this inhibitory effect was completely blocked by G-CSF. The mechanism by which DOX downregulates G-CSFR expression is entirely unknown, though DOX reportedly inhibits calcium-independent phospholipase $\mathrm{A}_{2}{ }^{40}$ It is thus tempting to speculate that inhibition of phospholipase $A_{2}$ by 
a
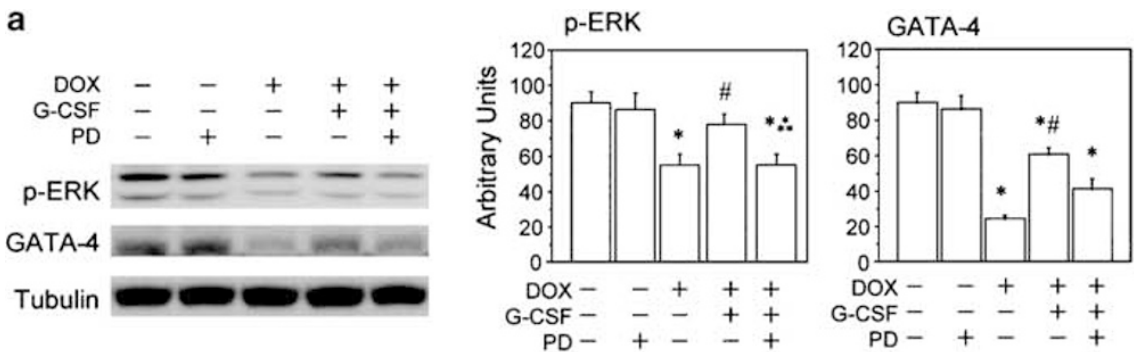

b

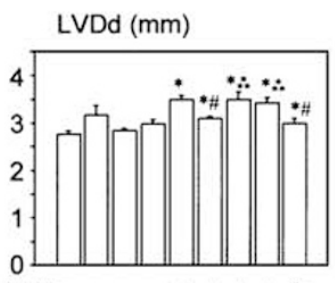

$\mathrm{DOX}----+++++$ G-CSF - - - - + + + +

$\mathrm{PD}-+--1+-$

NS --+---+

AMD - - +---+

$\operatorname{LVEDP}(\mathrm{mm} \mathrm{Hg})$

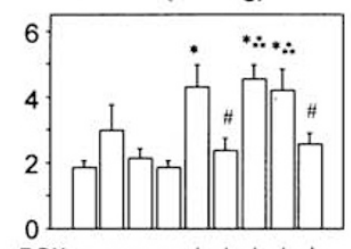

DOX ----+++++

G-CSF - - - - + + +

$\mathrm{PD}-+---+-1$

NS --+---+

C

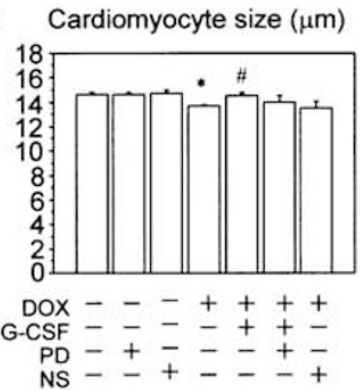

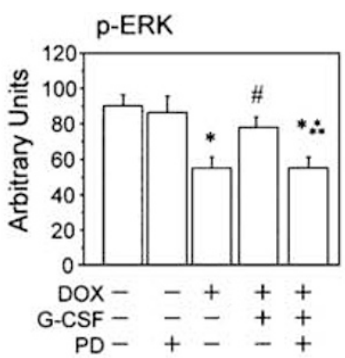

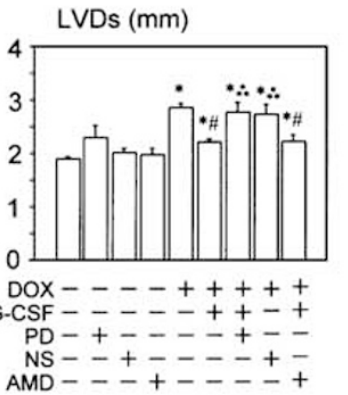

(dP/dt (mm Hig/sec)

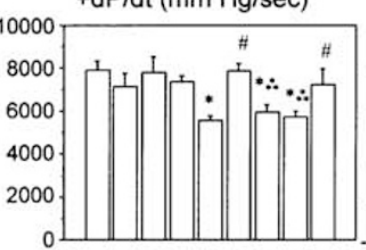

$\mathrm{DOX}----+++++$ G-CSF - - - - + + - +

$\mathrm{PD}-+---+--$

NS --+---+

AMD ---+---+

\%Fibrosis

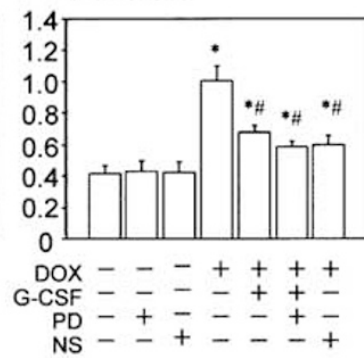

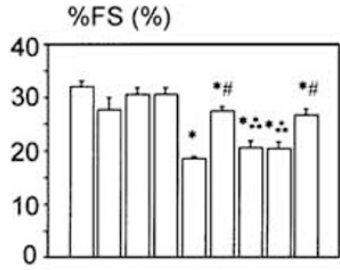

DOX - - - +++++ G-CSF -----+++

$\mathrm{PD}-+---+-1$

$\mathrm{PD}-+-\overline{-}+-\overline{-}$

AMD - - $+---1+$

$-\mathrm{dP} / \mathrm{dt}(\mathrm{mm} \mathrm{Hg} / \mathrm{sec})$

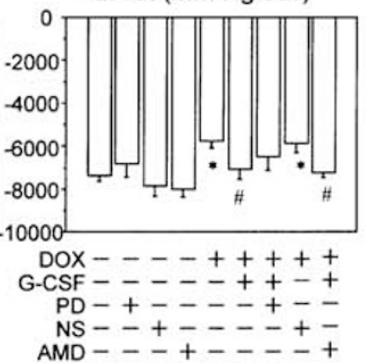

CD $45^{+}$Cells (/HPF)

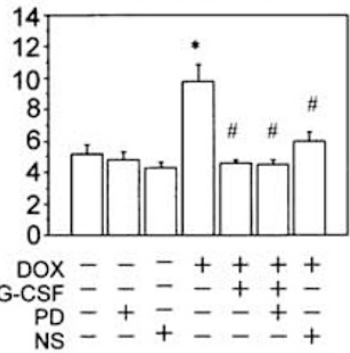

DOX
G-CSF
PD

$\operatorname{cox}-2$

Tubulin
$\mathrm{NS}--+---+$

Figure 8 (a) Western analysis of the effect of the p42/p44 MAPK inhibitor PD98059 (PD) on myocardial p-ERK and GATA-4 expressions. (b and c) Effect of PD, the COX-2 inhibitor NS398 (NS), and AMD3100 (AMD) on DOX-induced cardiomyopathy. Bars are means \pm s.e.m.; ${ }^{\star} P<0.05$ vs the untreated sham group (DOX (-) and G-CSF $(-)) ;{ }^{*} P<0.05$ vs the untreated DOX group (DOX $(+)$ and G-CSF $(-)) ;{ }_{* \star *}^{*} P<0.05$ vs the G-CSF-treated DOX group (DOX (+) and GCSF $(+))$. (d) Western blot analysis and the densitometries of the effect of NS on myocardial p-ERK and GATA-4 expressions. NS does not affect inhibition of either p-ERK or GATA-4 by DOX. (e) Western analysis and the densitometry of the effect of PD on myocardial COX-2 expression. PD does not restore inhibition of COX-2 by G-CSF.
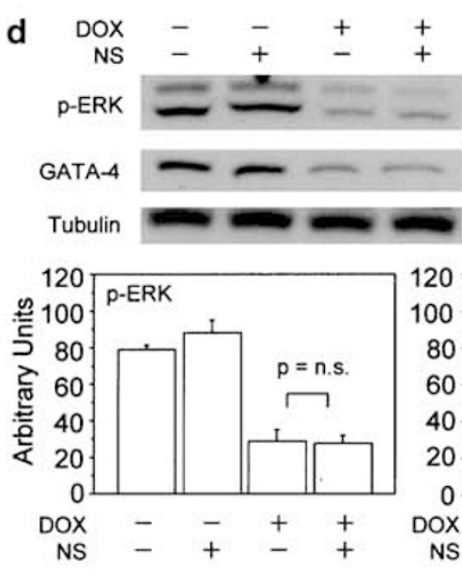

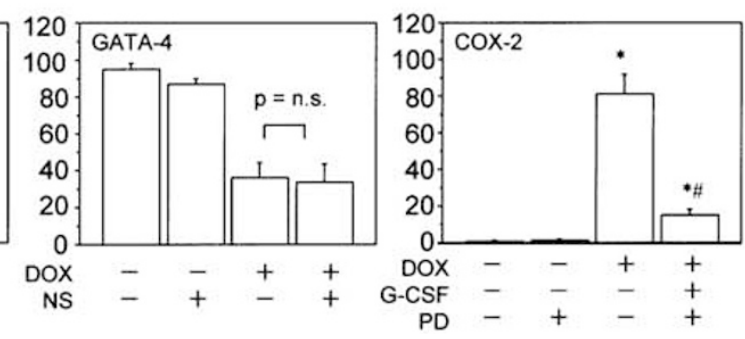



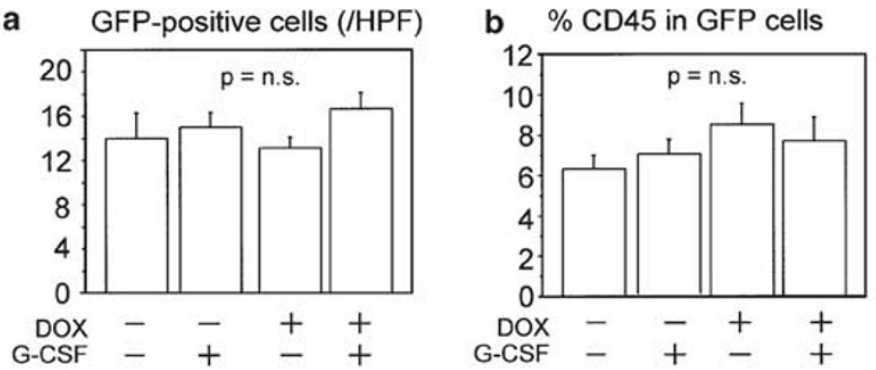

C
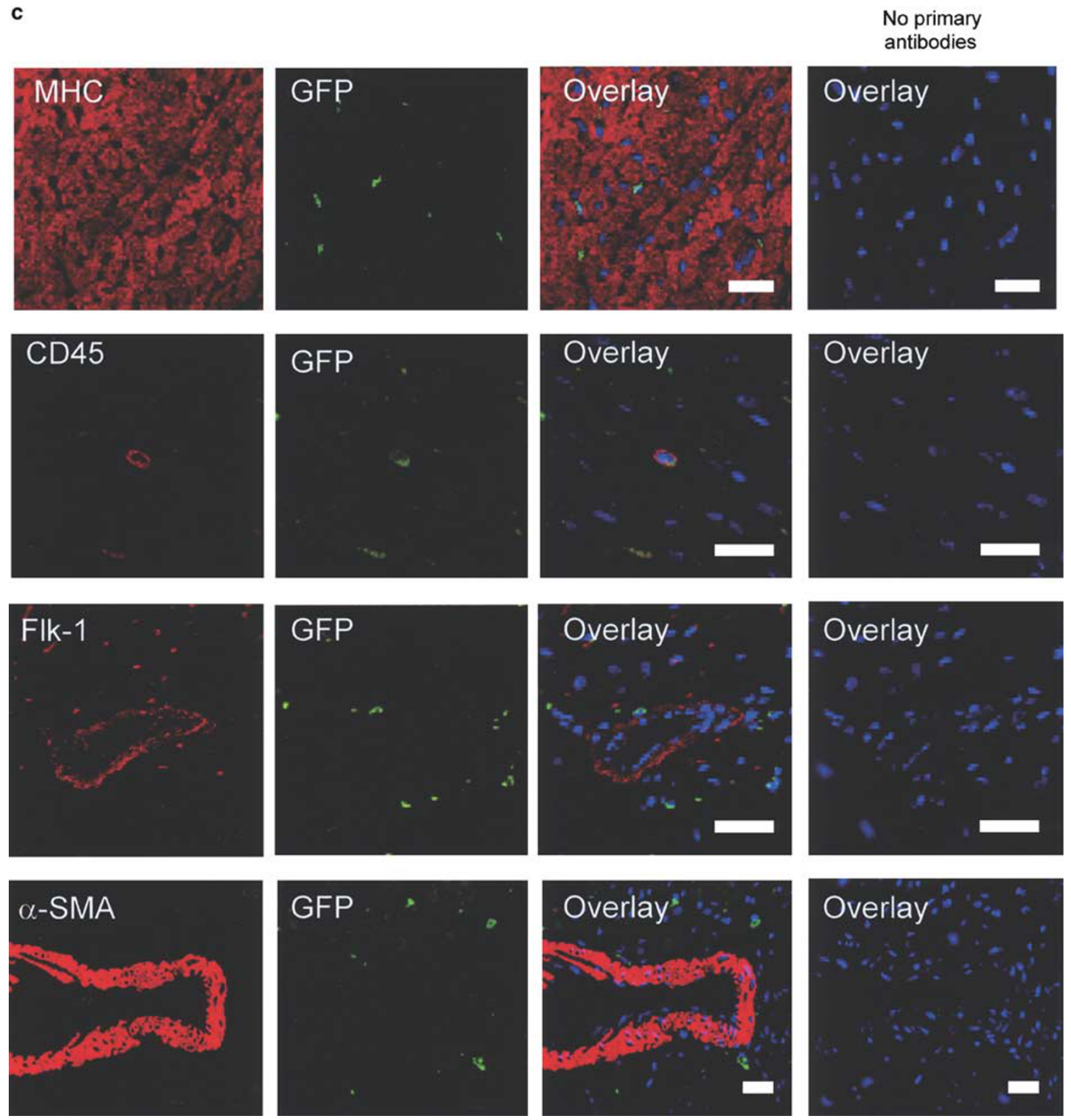

Figure 9 (a) Graphs showing the number of migrated bone marrow-derived GFP-positive cells in hearts and the percentage of myocardial CD45-positive cells among those originating from bone marrow-derived cells. (b) Confocal micrographs showing double immunofluorescent labeling of GFP with MHC, CD45, Flk-1 or $\alpha$-SMA. GFP positivity (green fluorescence) overlapped with CD45-positive leukocytes (red fluorescence), but not with cardiomyocytes, endothelial cells or vascular smooth muscle cells. When the primary antibodies were substituted with the control lgG, no fluorescence was noted of GFP or the cell specific proteins, confirming specificities of staining (the most right panels). Bars, $20 \mu \mathrm{m}$. 
Table 1 Left ventricular geometry, function and histology 4 weeks after administering DOX to mice with and without G-CSF (Protocol 2a)

\begin{tabular}{lccl}
\hline & DOX $(n=9)$ & DOX+G-CSF $(n=8)$ & $P$-value \\
\hline LVDd (mm) & $3.5 \pm 0.08$ & $3.3 \pm 0.11$ & $0.0913(\mathrm{NS})$ \\
LVDs (mm) & $2.8 \pm 0.10$ & $2.5 \pm 0.12$ & $0.0391^{*}$ \\
\%FS, (\%) & $20 \pm 1.1$ & $25 \pm 1.8$ & $0.0332^{*}$ \\
LVEDP (mm Hg) & $4.5 \pm 0.59$ & $2.7 \pm 0.48$ & $0.0471^{*}$ \\
LVSP (mm Hg) & $74 \pm 3.7$ & $91 \pm 4.3$ & $0.0123^{*}$ \\
Heart Rate (bpm) & $470 \pm 32$ & $528 \pm 35$ & $0.2467(\mathrm{NS})$ \\
$+\mathrm{dP} / \mathrm{d} t(\mathrm{~mm} \mathrm{Hg} / \mathrm{s})$ & $5528 \pm 335$ & $6520 \pm 291$ & $0.0482^{*}$ \\
$-\mathrm{dP} / \mathrm{d} t(\mathrm{~mm} \mathrm{Hg} / \mathrm{s})$ & $-5148 \pm 526$ & $-7582 \pm 295$ & $0.0023^{*}$ \\
Myocyte size $(\mu \mathrm{m})$ & $13.4 \pm 0.28$ & $14.3 \pm 0.26$ & $0.0399^{*}$ \\
$\%$ Fibrosis & $1.15 \pm 0.09$ & $0.57 \pm 0.08$ & $0.0004^{*}$ \\
CD45 $(\mathrm{cell} / \mathrm{HPF})$ & $12 \pm 1.3$ & $6.6 \pm 0.92$ & $0.0073^{*}$ \\
\hline
\end{tabular}

${ }^{*} P<0.05$.

DOX critically impairs plasma membrane function, which in turn inhibits insertion of G-CSFR into the cardiomyocyte plasma membrane.

Recent findings suggest that apoptosis among cardiomyocytes is a leading cause of cardiac dysfunction in DOXinduced cardiomyopathy, ${ }^{41}$ although this hypothesis is still controversial, because of the lack of evidence of apoptotic morphology in those cardiomyocytes. ${ }^{25,42}$ Hou et al attributed anti-apoptotic effect of G-CSF in DOX-induced cardiomyopathy to the beneficial mechanism. ${ }^{8}$ However, they depended on the TUNEL assay to detect apoptosis of which incidence was too high (more than 13\% of the cardiomyocytes) to approve and they failed in demonstrating apoptotic morphology in their electron microscopic analysis. ${ }^{8}$ Seeking evidence of DOX-induced apoptosis/cell death, we conducted a series of Evans blue living stains, TUNEL assays, electron microscopic examinations, analyses of myocardial caspase-3 activation, but we detected no effect of DOX or G-CSF on the incidence of apoptosis/cell death. Our findings thus suggest that cardiomyocyte apoptosis is not important for disease progression in the present model. The present model was given DOX in a single time and the survival rate was $100 \%$ in each group. These suggest that DOX insult in our model may be too weak to induce death in cardiac cells, compared with the previous ones. This may hold true also in our in vitro model.

\section{Study Limitations}

Generated reactive oxygen species (ROS) and calcium overload are believed to be most important mediators of DOX toxicity to the heart, ${ }^{1-3,41}$ although many other mechanisms are also proposed. These mediators are presumed to be positioning most upstream among signals evoked by DOX. The
Table 2 Left ventricular geometry and function 10 weeks after administering DOX to mice with and without G-CSF (Protocol 2b)

\begin{tabular}{lccc}
\hline & Sham $(n=6)$ & DOX $(n=10)$ & $\begin{array}{c}\text { DOX+G-CSF } \\
(n=10)\end{array}$ \\
\hline LVDd (mm/g) body & $0.141 \pm 0.005$ & $0.175 \pm 0.010^{*}$ & $0.156 \pm 0.005$ \\
weight ${ }^{\mathrm{a}}$ & & & \\
LVDs $(\mathrm{mm} / \mathrm{g})$ body & $0.101 \pm 0.005$ & $0.142 \pm 0.010^{*}$ & $0.115 \pm 0.005^{\#}$ \\
weight $^{\mathrm{a}}$ & & & \\
\%FS $(\%)$ & $28 \pm 1.2$ & $19 \pm 1.5^{*}$ & $24 \pm 1.2^{*, \#}$ \\
LVEDP $(\mathrm{mm} \mathrm{Hg})$ & $1.9 \pm 0.49$ & $3.9 \pm 0.46^{*}$ & $2.5 \pm 0.30^{\#}$ \\
LVSP $(\mathrm{mm} \mathrm{Hg})$ & $91 \pm 3.5$ & $89 \pm 2.6$ & $95 \pm 2.5$ \\
Heart rate, (bpm) & $556 \pm 19$ & $499 \pm 10^{*}$ & $488 \pm 14^{*}$ \\
$+\mathrm{d} P / \mathrm{d} t(\mathrm{~mm} \mathrm{Hg} / \mathrm{s})$ & $7266 \pm 340$ & $5931 \pm 295^{*}$ & $6843 \pm 288^{\#}$ \\
$-\mathrm{d} P / \mathrm{d} t(\mathrm{~mm} \mathrm{Hg} / \mathrm{s})$ & $-7890 \pm 418$ & $-6250 \pm 283^{*}$ & $-7460 \pm 425^{\#}$ \\
\hline
\end{tabular}

Significant difference with $P<0.05$ compared with the sham group ${ }^{*}$ ) or with the DOX only group $(\#)$.

aLVDd and LVDs were corrected with the body weight because the DOXtreated mice were under-grown.

present study, however, did not address the possible influence of G-CSF on them. Investigations of this interesting topic are warranted.

Phenotypes and molecular signals evoked by G-CSF here were strikingly similar to those by erythropoietin in our previous study using the same animal model of DOX-induced cardiomyopathy that is, anti-atrophic degeneration of cardiomyocytes, anti-fibrosis, restoration of ERK, GATA-4, and sarcomeric proteins, and inhibition of cyclooxygenase-2, although we did not note a therapeutic effect in case of erythropoietin. ${ }^{6}$ Although the intervention is different, many of the findings are quite similar. Thus, some comparative analysis between them is needed in future.

G-CSF has been reported to be angiogenic, ${ }^{12-14}$ but we detected no DOX-induced reduction in capillary density, nor did G-CSF promote capillary outgrowth, indicating no mechanistic role for angiogenesis in DOX-induced cardiomyopathy or the cardioprotective effects of G-CSF. G-CSF also reportedly enhances postinfarct myocardial regeneration by mobilizing bone marrow-derived cells to the myocardium. ${ }^{11,12}$ In the present model, however, we did not find that G-CSF promoted differentiation of bone marrow-derived cells into cardiomyocytes, endothelial cells or vascular smooth muscle cells. Moreover, inhibition of CXCR4 ${ }^{+}$cell homing by AMD3100 did not diminish the beneficial effect of G-CSF on cardiac function. Apparently, myocardial regeneration contributes little, if anything, to the effect of GCSF on DOX-induced cardiomyopathy. However, the present study did not address myocardial regeneration by resident cardiac stem cells $s^{43}$ and a possible effect of G-CSF on it remains unresolved. 
a
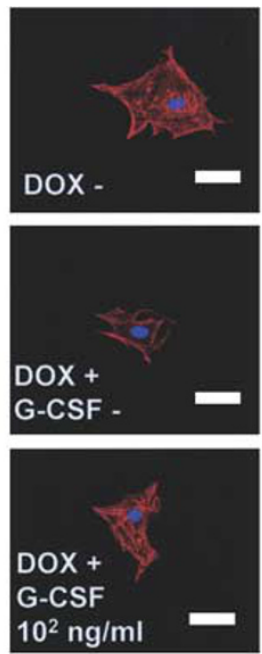

\section{Cardiomyocyte Area}

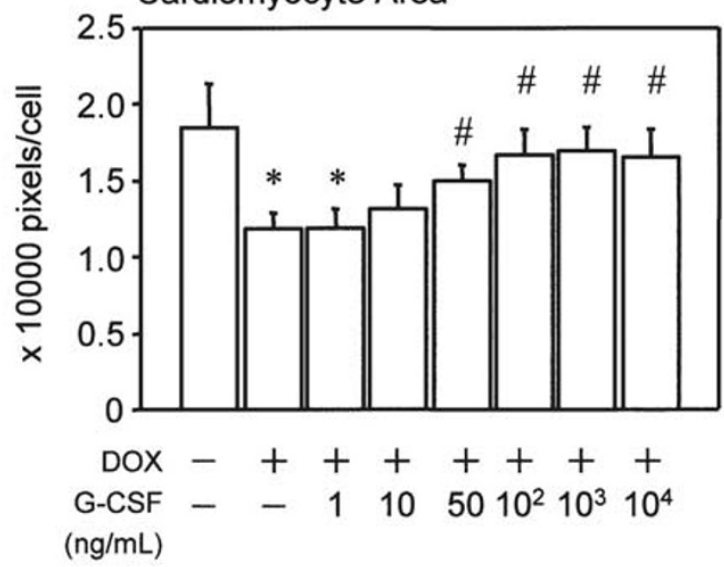

Figure 10 In vitro experiments (Protocol 3). (a) Confocal micrographs showing the atrophic degeneration of cardiomyocytes exposed to DOX and its prevention by GCSF. Bars, $10 \mu \mathrm{m}$. Graph shows the dosedependent effect of G-CSF on the size of cardiomyocytes exposed to DOX. (b and c) Western analysis of the effect of G-CSF on the DOX-mediated reduction in GATA4 and MHC expression (b) and increase in COX-2 expression (c) in cultured cardiomyocytes. Bars are means \pm s.e.m.; ${ }^{*} P<0.05$ vs the untreated sham group (DOX (-) and G-CSF (-)); ${ }^{\#} P<0.05$ vs the untreated DOX group (DOX $(+)$ and G-CSF (-)).

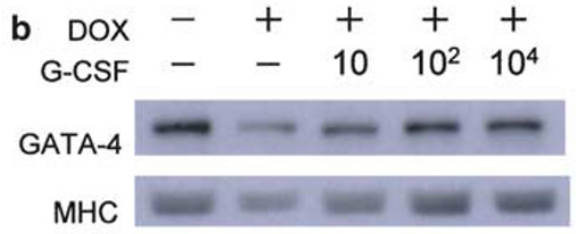

Tubulin
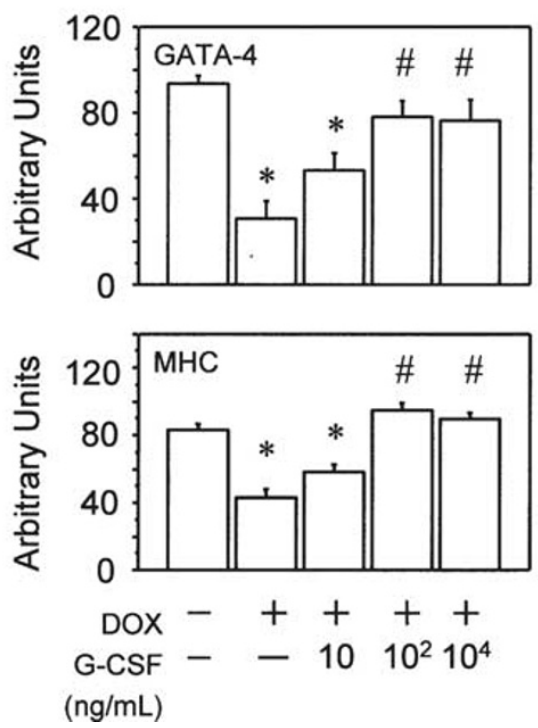

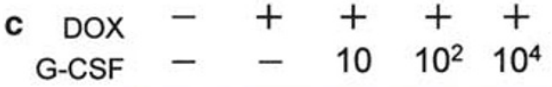

coX-2

Tubulin

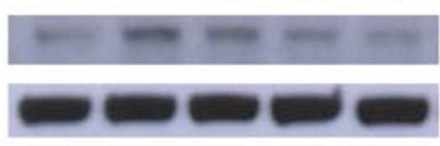

\section{Conclusions}

The present study not only confirms preventive effects, but also provides the first evidence of therapeutic effects of G-CSF against DOX-induced cardiomyopathy. These effects include attenuation of atrophic degeneration of cardiomyocytes and reduction of myocardial fibrosis, accompanied by restoration of myocardial expression of GATA-4 and sarcomeric proteins and suppression of COX-2 expression. Inhibition studies suggested that activated ERK-mediated antiatrophic degeneration effect might be the most important mechanism for the cardioprotection by G-CSF.

\section{ACKNOWLEDGEMENT}

We thank Akiko Tsujimoto and Hatsue Ohshika for technical assistance.

1. Olson RD, Mushlin P. Doxorubicin cardiotoxicity: analysis of prevailing hypotheses. FASEB J 1990;4:3076-3086.

2. Singal PK, lliskovic N. Doxorubicin-induced cardiomyopathy. N Engl J Med 1998;339:900-905.

3. Minotti G, Menna P, Salvatorelli E, et al. Anthracyclines: molecular advances and pharmacologic developments in antitumor activity and cardiotoxicity. Pharmacol Rev 2004;56:185-229.

4. Simpson C, Herr H, Courville KA. Concurrent therapies that protect against doxorubicin-induced cardiomyopathy. Clin J Oncol Nurs 2004;8:497-501. 
5. Sparano JA. Use of dexrazoxane and other strategies to prevent cardiomyopathy associated with doxorubicin-taxane combinations. Semin Oncol 1998;125(Suppl 10):66-71.

6. Li L, Takemura G, Li Y, et al. Preventive effect of erythropoietin on cardiac dysfunction in doxorubicin-induced cardiomyopathy.

Circulation 2006;113:535-543.

7. Li K, Sung RY, Huang WZ, et al. Thrombopoietin protects against in vitro and in vivo cardiotoxicity induced by doxorubicin. Circulation 2006;113:2211-2220.

8. Hou XW, Son J, Wang Y, et al. Granulocyte colony-stimulating factor reduces cardiomyocyte apoptosis and improves cardiac function in adriamycin-induced cardiomyopathy in rats. Cardiovasc Drugs Ther 2006:20:85-91.

9. Demetri GD, Griffin JD. Granulocyte colony-stimulating factor and its receptor. Blood 1991;78:2791-2808.

10. Avalos BR. Molecular analysis of the granulocyte colony-stimulating factor receptor. Blood 1996;88:761-777.

11. Orlic D, Kajstura J, Chimenti S, et al. Mobilized bone marrow cells repair the infarcted heart, improving function and survival. Proc Natl Acad Sci USA 2001;98:10344-10349.

12. Minatoguchi S, Takemura G, Chen XH, et al. Acceleration of the healing process and myocardial regeneration may be important as a mechanism of improvement of cardiac function and remodeling by postinfarction granulocyte colony-stimulating factor treatment. Circulation 2004;109:2572-2580.

13. Ohtsuka M, Takano $H$, Zou $Y$, et al. Cytokine therapy prevents left ventricular remodeling and dysfunction after myocardial infarction through neovascularization. FASEB J 2004;18:851-853.

14. Harada M, Qin $Y$, Takano $H$, et al. G-CSF prevents cardiac remodeling after myocardial infarction by activating the Jak-Stat pathway in cardiomyocytes. Nat Med 2005;11:305-311.

15. Cuenda A, Alessi DR. Use of kinase inhibitors to dissect signaling pathways. Methods Mol Biol 2000;99:161-175.

16. LaPointe MC, Mendez M, Leung A, et al. Inhibition of cyclooxygenase-2 improves cardiac function after myocardial infarction in the mouse. Am J Physiol Heart Circ Physiol 2004;286:H1416-H1424.

17. Okabe $M$, lkawa $M$, Kominami $K$, et al. Green mice as a source of ubiquitous green cells. FEBS Lett 1997;407:313-319.

18. Kushida $\mathrm{T}$, Inaba $\mathrm{M}$, Hisha $\mathrm{H}$, et al. Intra-bone marrow injection of allogeneic bone marrow cells: a powerful new strategy for treatment of intractable autoimmune diseases in MRL/lpr mice. Blood 2001;97:3292-3299.

19. Liles WC, Broxmeyer HE, Rodger E, et al. Mobilization of hematopoietic progenitor cells in healthy volunteers by AMD3100, a CXCR4 antagonist. Blood 2003;102:2728-2730.

20. Aoyama T, Takemura G, Maruyama R, et al. Molecular mechanisms of non-apoptosis by Fas stimulation alone vs apoptosis with an additional actinomycin D in cultured cardiomyocytes. Cardiovasc Res 2002;55:787-798.

21. Li Y, Takemura G, Okada H, et al. Treatment with granulocyte colonystimulating factor ameliorates chronic heart failure. Lab Invest 2006:86:32-44.

22. Kim Y, Ma A, Kitta K, et al. Anthracycline-induced suppression of GATA4 transcription factor: implication in the regulation of cardiac myocyte apoptosis. Mol Pharmacol 2003;63:368-377.

23. Aries A, Paradis P, Lefebvre $C$, et al. Essential role of GATA-4 in cell survival and drug-induced cardiotoxicity. Proc Natl Acad Sci USA 2004;101:6975-6980.

24. Kawada T, Nakazawa M, Nakauchi S, et al. Rescue of hereditary form of dilated cardiomyopathy by rAAV-mediated somatic gene therapy: amelioration of morphological findings, sarcolemmal permeability, cardiac performances, and the prognosis of TO-2 hamsters. Proc Natl Acad Sci USA 2002;99:901-906.
25. Rosenoff $\mathrm{SH}$, Olson HM, Young DM, et al. Adriamycin-induced cardiac damage in the mouse: a small-animal model of cardiotoxicity. J Natl Cancer Inst 1975:55:191-194.

26. Molkentin JD, Kalvakolanu DV, Markham BE. Transcription factor GATA4 regulates cardiac muscle-specific expression of the alpha-myosin heavy-chain gene. Mol Cell Biol 1994;14:4947-4957.

27. Murphy AM, Thompson WR, Peng LF, et al. Regulation of the rat cardiac troponin I gene by the transcription factor GATA-4. Biochem J 1997;322:393-401.

28. Rausch O, Marshall CJ. Cooperation of p38 and extracellular signalregulated kinase mitogen-activated protein kinase pathways during granulocyte colony-stimulating factor-induced hemopoietic cell proliferation. J Biol Chem 1999;274:4096-4105.

29. Hermans MH, Geijn GJ, Antonissen C, et al. Signaling mechanisms coupled to tyrosines in the granulocyte colony-stimulating factor receptor orchestrate G-CSF-induced expansion of myeloid progenitor cells. Blood 2003;101:2584-2590.

30. Lou H, Danelisen I, Singal PK. Involvement of mitogen-activated protein kinases in adriamycin-induced cardiomyopathy. Am J Physiol Heart Circ Physiol 2005;288:H1925-H1930.

31. Tenhunen O, Sarman B, Kerkela R, et al. Mitogen-activated protein kinases p38 and ERK 1/2 mediate the wall stress-induced activation of GATA-4 binding in adult heart. J Biol Chem 2004;279:24852-24860.

32. Østergaard C, Benfield T, Gesser B, et al. Pretreatment with granulocyte colony-stimulating factor attenuates the inflammatory response but not the bacterial load in cerebrospinal fluid during experimental pneumococcal meningitis in rabbits. Infect Immun 1999;67:3430-3436.

33. Boneberg EM, Hartung T. Molecular aspects of anti-inflammatory action of G-CSF. Inflamm Res 2002;51:119-128.

34. Wong SC, Fukuchi M, Melnyk $\mathrm{P}$, et al. Induction of cyclooxygenase-2 and activation of nuclear factor- $\kappa \mathrm{B}$ in myocardium of patients with congestive heart failure. Circulation 1998;98:100-103.

35. Bryant D, Becker L, Richardson J, et al. Cardiac failure in transgenic mice with myocardial expression of tumor necrosis factor-alpha. Circulation 1998;97:1375-1381.

36. Hein S, Arnon E, Kostin S, et al. Progression from compensated hypertrophy to failure in the pressure-overloaded human heart: structural deterioration and compensatory mechanisms. Circulation 2003;107:984-991.

37. Lou H, Danelisen I, Singal PK. Cytokines are not upregulated in adriamycin-induced cardiomyopathy and heart failure. J Mol Cell Cardiol 2004;36:683-690.

38. Adderley SR, Fitzgerald DJ. Oxidative damage of cardiomyocytes is limited by extracellular regulated kinases 1/2-mediated induction of cyclooxygenase-2. J Biol Chem 1999;274:5038-5046.

39. Delgado III RM, Nawar MA, Zewail AM, et al. Cyclooxygenase-2 inhibitor treatment improves left ventricular function and mortality in a murine model of doxorubicin-induced heart failure. Circulation 2004;109:1428-1433.

40. Swift L, McHowat J, Sarvazyan N. Inhibition of membrane-associated calcium-independent phospholipase $A_{2}$ as a potential culprit of anthracycline cardiotoxicity. Cancer Res 2003;63:5992-5998.

41. Kalyanaraman B, Joseph J, Kalivendi S, et al. Doxorubicin-induced apoptosis: implications in cardiotoxicity. Mol Cell Biochem 2002;234235:119-124.

42. Zhang J, Clark Jr JR, Herman EH, et al. Doxorubicin-induced apoptosis in spontaneously hypertensive rats: differential effects in heart, kidney and intestine, and inhibition by ICRF-187. J Mol Cell Cardiol 1996;28:1931-1943.

43. Mendez-Ferrer S, Ellison GM, Torella D, et al. Resident progenitors and bone marrow stem cells in myocardial renewal and repair. Nat Clin Pract Cardiovasc Med 2006;3(Suppl 1):S83-S89. 